\title{
Quantum graphs with singular two-particle interactions
}

\author{
Jens Bolte1 and Joachim Kerner² \\ Department of Mathematics \\ Royal Holloway, University of London \\ Egham, TW20 0EX \\ United Kingdom
}

\begin{abstract}
We construct quantum models of two particles on a compact metric graph with singular two-particle interactions. The Hamiltonians are self-adjoint realisations of Laplacians acting on functions defined on pairs of edges in such a way that the interaction is provided by boundary conditions. In order to find such Hamiltonians closed and semi-bounded quadratic forms are constructed, from which the associated self-adjoint operators are extracted. We provide a general characterisation of such operators and, furthermore, produce certain classes of examples. We then consider identical particles and project to the bosonic and fermionic subspaces. Finally, we show that the operators possess purely discrete spectra and that the eigenvalues are distributed following an appropriate Weyl asymptotic law.
\end{abstract}

\footnotetext{
${ }^{1}$ E-mail address: jens.bolte@rhul.ac.uk

${ }^{2}$ E-mail address: joachim.kerner.2010@live.rhul.ac.uk
} 


\section{Introduction}

Initially, quantum graphs were introduced by Ruedenberg and Scherr [RS53] as simplified models to describe the electronic structure of aromatic hydrocarbons, where the graph connectivity follows from the bond structure of the atoms in the molecule. Since then numerous variants of quantum graphs have been developed and have found applications in many areas of physics and mathematics (see [EKK ${ }^{+} 08$ for a review).

Quantum systems on graphs have proven to be attractive and versatile models as they combine the simplicity of one (or zero) spatial dimension with the complexity induced by the connectivity of the underlying graph. In the following we shall focus on models in which one-dimensional quantum systems associated with the edges of a (metric) graph are coupled in the vertices of the graph. Alternative models utilise finite-dimensional quantum systems associated with the (zero-dimensional) vertices of a graph.

In mathematical terms, the stationary Schrödinger equation of a quantum graph is a system of coupled, ordinary differential equations, describing the quantum motion on the edges of the graph that are connected in the vertices. Quite surprisingly, such coupled systems of equations can be used to interpolate between single, ordinary differential equations describing pure, one-dimensional quantum systems on the one hand, and partial differential equations as they arise in multi-dimensional quantum systems on the other hand. Indeed, Kottos and Smilansky observed that the eigenvalue correlations in quantum graphs generically are the same as in quantum systems with chaotic classical limit [KS99b]. The latter require at least two spatial dimensions and thus are modelled by partial differential equations. Following the random matrix conjecture of quantum chaos [BGS84, these correlations can be described by eigenvalue correlations of suitable random matrices. Many further studies along these lines have followed since (see [GS06] for a review).

So far quantum graphs mostly have been used to model one-particle systems. Harmer, however, introduced $\delta$-type two-particle interactions on the edges [Har07, Har08], and Harrison et al. studied the particle exchange symmetry in many-particle versions of finitedimensional quantum graph models [HKR11]. In this paper we shall study two-particle quantum systems on graphs. Our focus will be the introduction of genuine two-particle interactions along the lines of the one-particle interactions (with the outside world) present in the existing quantum graph models. The latter represent singular interactions ("potentials') that are strictly localised in the vertices of the graph so that the motion along edges is free. The associated quantum Hamiltonians are constructed as self-adjoint extensions of a symmetric realisation of the Laplacian acting on functions on the edges. These extensions introduce coupling conditions in the vertices and hence provide localised interactions. Following the tensor-product construction of many-particle quantum models, two-particle states on graphs are composed of functions on pairs of edges, one for each particle, and the free Hamiltonian is a Laplacian acting on functions defined on rectangles. The natural, symmetric realisation of such a Laplacian, however, has infinite deficiency indices so that a construction of self-adjoint extensions is less straight forward than in the one-particle case. Therefore, we first define suitable, closed and semi-bounded quadratic forms and then extract from such a form the unique, semi-bounded and self-adjoint operator that 
is associated with it. This operator is, of course, again a Laplacian acting on functions on rectangles, but with boundary conditions imposed along the edges of the rectangles. In a general setting these boundary conditions emerge in a weak form, but under specific circumstances related to elliptic regularity we are able to provide explicit versions of these conditions that are closely related to the respective conditions in a one-particle quantum graph. The resulting two-particle interactions are singular and, in contrast to Harmer's construction [Har07, Har08, localised in vertices rather than on edges.

Our main results are, first, the construction of self-adjoint operators that describe singular two-particle interactions on graphs and, second, proving that these operators possess purely discrete spectra and that their eigenvalue count follows a suitable Weyl asymptotic law.

This paper is organised as follows: In Section 2 we briefly recall the construction of one-particle quantum graphs as well as some of their properties that are relevant for our purposes. Then we describe the tensor-product construction of systems of two distinguishable as well as of two identical particles as applied to quantum graphs. In Section 3 we first perform the construction of self-adjoint operators for two distinguishable particles on an interval via a suitable quadratic form. We then extend these constructions to arbitrary compact metric graphs. We also characterise those among the self-adjoint operators that represent non-trivial two-particle interactions. The necessary adaptions to systems of two identical particles (bosons or fermions) on general compact graphs are performed in Section 4. In Section 5 we prove that the two-particle Hamiltonians constructed before have compact resolvent and, therefore, possess purely discrete spectra; their eigenvalue count follows a Weyl-type asymptotic law. We defer the proof our main regularity result in Section 3 to an appendix.

\section{Preliminaries}

Quantum systems with many particles are obtained from tensor product constructions based on the underlying one-particle systems. For that reason we briefly summarise oneparticle quantum graphs, and then explain the basic steps to construct quantum systems of two (identical) particles on a graph. For more details on one-particle quantum graphs see [KS99b, KS99a, Kuc04, GS06, EKK ${ }^{+} 08$, BE09].

\subsection{One-particle quantum graphs}

The classical configuration space of a quantum graph is a compact metric graph, i.e., a finite graph $\Gamma=(\mathcal{V}, \mathcal{E})$ with vertices $\mathcal{V}=\left\{v_{1}, \ldots, v_{V}\right\}$ and edges $\left\{e_{1}, \ldots, e_{E}\right\}$. The latter are identified with intervals $\left[0, l_{e}\right], e=1, \ldots, E$, thus introducing a metric on the graph. At this point we do not exclude multiple edges and loops.

Functions on the graph are collections of functions on the edges, i.e.,

$$
F=\left(f_{1}, \ldots, f_{E}\right), \quad \text { with } \quad f_{e}:\left[0, l_{e}\right] \rightarrow \mathbb{C},
$$


so that spaces of functions on $\Gamma$ are (finite) direct sums of the respective spaces of functions on the edges. The most relevant space is the one-particle Hilbert space

$$
\mathcal{H}_{1}=L^{2}(\Gamma):=\bigoplus_{e=1}^{E} L^{2}\left(0, l_{e}\right),
$$

and all other spaces are constructed in a similar way.

One-particle observables are self-adjoint operators on $\mathcal{H}_{1}$, among which the Hamiltonian plays a prominent role. In the absence of external forces or gauge fields the Hamiltonian should be a suitable version of a Laplacian. As a differential operator the (positive) Laplacian acts according to

$$
-\Delta_{1} F=\left(-f_{1}^{\prime \prime}, \ldots,-f_{E}^{\prime \prime}\right)
$$

on $F \in C^{\infty}(\Gamma)$. We here use the index to indicate that this is a one-particle Laplacian.

Viewed as an operator in $L^{2}(\Gamma)$ with domain $C_{0}^{\infty}(\Gamma)$, this Laplacian is symmetric, but not self-adjoint. One can construct and classify all self-adjoint extensions of this operator using von Neumann's theory. In the context of quantum graphs, however, an alternative parametrisation of self-adjoint extensions in terms of linear relations among the boundary values

$$
F_{b v}:=\left(f_{1}(0), \ldots, f_{E}(0), f_{1}\left(l_{1}\right), \ldots, f_{E}\left(l_{E}\right)\right)^{T} \in \mathbb{C}^{2 E},
$$

of functions and (inward) derivatives,

$$
F_{b v}^{\prime}:=\left(f_{1}^{\prime}(0), \ldots, f_{E}^{\prime}(0),-f_{1}^{\prime}\left(l_{1}\right), \ldots,-f_{E}^{\prime}\left(l_{E}\right)\right)^{T} \in \mathbb{C}^{2 E}
$$

has proven useful. Kostrykin and Schrader [KS99a] proved the following.

Theorem 2.1 (Kostrykin, Schrader). Any self-adjoint realisation of the Laplacian on a compact, metric graph has a domain of the form

$$
\mathcal{D}_{1}(A, B)=\left\{F \in H^{2}(\Gamma) ; A F_{b v}+B F_{b v}^{\prime}=0\right\},
$$

where $A, B \in \mathrm{M}(2 E, \mathbb{C})$ are such that $\operatorname{rk}(A, B)=2 E$ and $A B^{*}$ is self-adjoint.

Moreover, two such realisations, with domains $\mathcal{D}(A, B)$ and $\mathcal{D}\left(A^{\prime}, B^{\prime}\right)$, are equivalent, iff there exists $C \in \mathrm{GL}(2 E, \mathbb{C})$ such that $A^{\prime}=C A$ and $B^{\prime}=C B$.

An alternative characterisation of the domain (2.6) employs the orthogonal projectors $P$ onto ker $B \subset \mathbb{C}^{2 E}$ and $Q=\mathbb{1}_{2 E}-P$, as well as the self-adjoint endomorphism $L=$ $\left(\left.B\right|_{\operatorname{ran} B^{*}}\right)^{-1} A Q$ of $\operatorname{ran} Q \subset \mathbb{C}^{2 E}$. Kuchment showed [Kuc04 that the domain $\mathcal{D}_{1}(A, B)$ is the same as

$$
\mathcal{D}_{1}(P, L)=\left\{F \in H^{2}(\Gamma) ; P F_{b v}=0 \text { and } Q F_{b v}^{\prime}+L Q F_{b v}=0\right\} .
$$

This way self-adjoint realisations of the Laplacian are uniquely characterised in terms of the projector $P$ and the self-adjoint map $L$. From now on we shall adhere to this parametrisation of domains.

Yet another way of specifying self-adjoint Laplacians is in terms of their associated quadratic forms [Kuc04. 
Theorem 2.2 (Kuchment). The quadratic form associated with a Laplacian $-\Delta_{1}$ defined on the domain $\mathcal{D}_{1}(P, L)$ is

$$
\begin{aligned}
Q_{P, L}^{(1)}[F] & =\int_{\Gamma}|\nabla f| \mathrm{d} x-\left\langle F_{b v}, L F_{b v}\right\rangle_{\mathbb{C}^{2 E}} \\
& =\sum_{e=1}^{E} \int_{0}^{l_{e}}\left|f_{e}^{\prime}(x)\right|^{2} d x-\left\langle F_{b v}, L F_{b v}\right\rangle_{\mathbb{C}^{2 E}},
\end{aligned}
$$

with form domain

$$
\mathcal{D}_{Q^{(1)}}=\left\{F \in H^{1}(\Gamma) ; P F_{b v}=0\right\} .
$$

In all of the above the boundary conditions imposed on functions in the domains are such that, in principle, they relate all boundary values among each other. Whenever the boundary conditions only relate boundary values at edge ends that are connected in a given vertex, the boundary conditions are said to be local. In that case the linear maps $A, B, P, Q, L$ are block-diagonal with respect to the decomposition

$$
\mathbb{C}^{2 E}=\bigoplus_{v \in \mathcal{V}} \mathbb{C}^{d_{v}}
$$

of the space of boundary values, where $d_{v}$ is the degree of the vertex $v$.

When boundary conditions are local one can view them as representing local, singular interactions of the particle on the graph with an 'external potential' in the vertices. Nonlocal boundary conditions would model non-local, singular forces acting on the particle and are, therefore, often discarded.

\subsection{Two-particle quantum graphs}

Placing two particles on a graph first requires to introduce a two-particle Hilbert space. For two distinguishable particles this is the tensor product of two one-particle Hilbert spaces,

$$
\mathcal{H}_{2}:=\mathcal{H}_{1} \otimes \mathcal{H}_{1}
$$

For a quantum graph this means that

$$
\mathcal{H}_{2}:=\left(\bigoplus_{e=1}^{E} L^{2}\left(0, l_{e}\right)\right) \otimes\left(\bigoplus_{e=1}^{E} L^{2}\left(0, l_{e}\right)\right)
$$

such that vectors $\Psi \in \mathcal{H}_{2}$ are collections $\Psi=\left(\psi_{e_{1} e_{2}}\right)$ of $E^{2}$ functions

$$
\psi_{e_{1} e_{2}} \in L^{2}\left(0, l_{e_{1}}\right) \otimes L^{2}\left(0, l_{e_{2}}\right) .
$$

Two-particle observables are self-adjoint operators in $\mathcal{H}_{2}$. By the above, these are given in components as,

$$
(O \Psi)_{e_{1} e_{2}}=\sum_{f_{1}, f_{2}=1}^{E} O_{e_{1} e_{2}, f_{1} f_{2}} \psi_{f_{1} f_{2}}
$$


A particular set of two-particle observables are those that are given as lifts of one-particle observables. If, for simplicity we restrict our attention to bounded operators, a one-particle observable $O_{1}$ can be lifted to a two-particle observable as

$$
O_{2}:=O_{1} \otimes \mathbb{1}_{\mathcal{H}_{1}}+\mathbb{1}_{\mathcal{H}_{1}} \otimes O_{1} .
$$

Unbounded operators allow for an equivalent construction (see [RS72]). Any observable of this kind does not represent interactions, or correlations, between the particles.

On a formal level, the one-particle Laplacian has an obvious lift to a two-particle operator $-\Delta_{2}$; its operator-matrix entries read

$$
-\Delta_{2, e_{1} e_{2}}=-\frac{\partial^{2}}{\partial x_{e_{1}}^{2}}-\frac{\partial^{2}}{\partial x_{e_{2}}^{2}},
$$

and hence have the same form as a Laplacian in $\mathbb{R}^{2}$. Defined on the domain $C_{0}^{\infty}(\Gamma) \otimes C_{0}^{\infty}(\Gamma)$, this operator is symmetric, but not self-adjoint. Again, as in the one-particle case, the selfadjoint extensions of this operator are observables, and hence candidates for a two-particle Hamiltonian.

Below we shall see that among the self-adjoint realisations of the two-particle Laplacian we can identify classes of operators that, indeed, are lifts of one-particle Laplacians, and others that are not. The latter represent genuine two-particle interactions and their identification and characterisation is the principal goal of this paper.

We also want to consider identical particles. This means that a particle exchange is a symmetry of the quantum system and hence the symmetric group $S_{2}$ has to be represented unitarily on the two-particle Hilbert space. Following the symmetrisation postulate for a system of $N$ identical particles, the two physically relevant irreducible representations of $S_{N}$ are the totally symmetric and the totally anti-symmetric representation; according to the spin-statistic theorem, these representations correspond to bosons and fermions, respectively. When $N=2$ these are the only unitary irreducible representations anyway. The bosonic, i.e., the totally symmetric representation is defined on the bosonic twoparticle Hilbert space $\mathcal{H}_{2, B}$, which is the symmetric tensor product of two one-particle spaces. Hence, $\Psi=\left(\psi_{e_{1} e_{2}}\right) \in \mathcal{H}_{2, B}$, iff

$$
\psi_{e_{1} e_{2}}\left(x_{e_{1}}, x_{e_{2}}\right)=\psi_{e_{2} e_{1}}\left(x_{e_{2}}, x_{e_{1}}\right) .
$$

The projection $\Pi_{s}: \mathcal{H}_{2} \rightarrow \mathcal{H}_{2, B}$ then reads

$$
\left(\Pi_{s} \Psi\right)_{e_{1} e_{2}}=\frac{1}{2}\left(\psi_{e_{1} e_{2}}+\psi_{e_{2} e_{1}}\right)
$$

Similarly, the fermionic, totally antisymmetric representation is defined on the fermionic two-particle Hilbert space $\mathcal{H}_{2, F}$, which is the anti-symmetric tensor product of $\mathcal{H}_{1}$ with itself. Accordingly, $\Psi=\left(\psi_{e_{1} e_{2}}\right) \in \mathcal{H}_{2, F}$, iff

$$
\psi_{e_{1} e_{2}}\left(x_{e_{1}}, x_{e_{2}}\right)=-\psi_{e_{2} e_{1}}\left(x_{e_{2}}, x_{e_{1}}\right) .
$$

The projection $\Pi_{a}: \mathcal{H}_{2} \rightarrow \mathcal{H}_{2, F}$ then reads

$$
\left(\Pi_{a} \Psi\right)_{e_{1} e_{2}}=\frac{1}{2}\left(\psi_{e_{1} e_{2}}-\psi_{e_{2} e_{1}}\right)
$$




\section{Two-particle interactions}

We now introduce singular two-particle interactions. In a first step we perform a detailed analysis of two distinguishable, interacting particles on an interval. This is then generalised to two distinguishable particles on a general compact, metric graph.

\subsection{Two distinguishable particles on an interval}

As a first step towards our principal goal we now address the most simple graph consisting of two vertices and one edge, i.e., an interval $[0, l]$. In that case all two-particle functions are defined on the square $D:=(0, l) \times(0, l)$. Hence, in particular, the Hilbert space for two distinguishable particles is $\mathcal{H}_{2}=L^{2}(0, l) \otimes L^{2}(0, l)=L^{2}(D)$.

The goal now is to find self-adjoint realisations of the Laplacian with a domain that is a subspace of $L^{2}(D)$. In the one-particle case self-adjoint realisations of the Laplacian were obtained as maximally symmetric extensions of a suitable symmetric realisation of the Laplacian. In the two-particle case the corresponding Laplacian $-\Delta_{2,0}$ has a domain $C_{0}^{\infty}(D)$. Hence, the domain of its adjoint $-\Delta_{2,0}^{*}$ is

$$
\mathcal{D}\left(-\Delta_{2,0}^{*}\right)=\left\{\psi \in L^{2}(D) ; \exists \chi \in L^{2}(D) \text { s.t. }\left\langle\psi,-\Delta_{2,0} \phi\right\rangle=\langle\chi, \phi\rangle \forall \phi \in C_{0}^{\infty}(D)\right\} .
$$

Notice here that in general $\mathcal{D}\left(-\Delta_{2,0}^{*}\right) \neq H^{2}(D)$, but $H^{2}(D) \subset \mathcal{D}\left(-\Delta_{2,0}^{*}\right)$. Whether this implies that a domain of a self-adjoint realisation of the Laplacian is in $H^{2}(D)$ is a subtle issue, related to the problem of (elliptic) regularity, and will be addressed in detail below.

Although the situation is similar to the one-particle case, one cannot proceed to classify self-adjoint extensions of $-\Delta_{2,0}$ in the same way as the deficiency indices of $-\Delta_{2,0}$ are infinite. It is, therefore, not automatically guaranteed that the maximally symmetric extensions of $-\Delta_{2,0}$ are self-adjoint, see [RS79].

Nevertheless, for the following it will be useful to generate a certain class of extensions of $-\Delta_{2,0}$. Their domains are subsets of $\mathcal{D}\left(-\Delta_{2,0}^{*}\right)$ and, in close analogy to the one-particle case, shall be characterised in terms of boundary conditions imposed on the functions. These will involve the boundary values (traces) of functions $\psi \in H^{1}(D)$ as well of their derivatives (in which case $\psi$ has to be in $H^{2}(D)$ ) that, for convenience, are arranged as follows,

$$
\psi_{b v}(y)=\left(\begin{array}{c}
\psi(0, y) \\
\psi(l, y) \\
\psi(y, 0) \\
\psi(y, l)
\end{array}\right) \quad \text { and } \quad \psi_{b v}^{\prime}(y)=\left(\begin{array}{c}
\psi_{x}(0, y) \\
-\psi_{x}(l, y) \\
\psi_{y}(y, 0) \\
-\psi_{y}(y, l)
\end{array}\right)
$$

i.e., as functions in $L^{2}(0, l) \otimes \mathbb{C}^{4}$. We also require maps $P, L:[0, l] \rightarrow \mathrm{M}(4, \mathbb{C})$ fulfilling

1. $P(y)$ is an orthogonal projector,

2. $L(y)$ is self-adjoint endomorphism of $\operatorname{ker} P(y)$, 
for a.e. $y \in[0, l]$. These maps shall be (at least) measurable and bounded.

With these maps, as well as with $Q(y):=\mathbb{1}_{4}-P(y)$, we define the domains

$$
\begin{aligned}
\mathcal{D}_{2}(P, L):=\{\psi \in & H^{2}(D) ; P(y) \psi_{b v}(y)=0 \text { and } \\
& \left.Q(y) \psi_{b v}^{\prime}(y)+L(y) Q(y) \psi_{b v}(y)=0 \text { for a.e. } y \in[0, l]\right\},
\end{aligned}
$$

that will be useful later on.

In the same way as for one-particle Laplacians, an equivalent characterisation in terms of maps $A, B:[0, l] \rightarrow \mathrm{M}(4, \mathbb{C})$ is available, see (2.6) vs. (2.7). These maps are required to fulfil for a.e. $y \in[0, l]$ that $\operatorname{rk}(A(y), B(y))=4$ and that $A(y) B(y)^{*}$ is self-adjoint. In that case $P(y)$ is a projector onto $\operatorname{ker} B(y) \subseteq \mathbb{C}^{4}$ and the self-adjoint map is given by $L(y)=$ $\left(\left.B(y)\right|_{\operatorname{ran} B(y)^{*}}\right)^{-1} A(y) Q(y)$ on $\mathbb{C}^{4}$; indeed, it is an endomorphisms of $\operatorname{ran} B(y)^{*}=\operatorname{ran} Q(y)$.

In a next step we generate a closed and semi-bounded quadratic form that allows us to define self-adjoint realisations of the two-particle Laplacian. Before, however, we introduce some useful notation: The maps $P, L:[0, l] \rightarrow \mathrm{M}(4, \mathbb{C})$ define (multiplication) operators $\Pi$ and $\Lambda$, respectively, on $L^{2}(0, l) \otimes \mathbb{C}^{4}$ through $(\Pi \chi)(y):=P(y) \chi(y)$ and $(\Lambda \chi)(y):=L(y) \chi(y)$, $\chi \in L^{2}(0, l) \otimes \mathbb{C}^{4}$. As the functions $P$ and $L$ are bounded and measurable on $[0, l]$, the operators $\Pi$ and $\Lambda$ are bounded; $\Pi$ is a projector and $\Lambda$ is self-adjoint.

The quadratic form then will derive from the sesqui-linear form

$$
\begin{aligned}
Q_{P, L}^{(2)}[\psi, \phi]:= & \langle\nabla \psi, \nabla \phi\rangle_{L^{2}(D)}-\left\langle\psi_{b v}, \Lambda \phi_{b v}\right\rangle_{L^{2}(0, l) \otimes \mathbb{C}^{4}} \\
= & \int_{0}^{l} \int_{0}^{l}\left(\overline{\psi_{x}(x, y)} \phi_{x}(x, y)+\overline{\psi_{y}(x, y)} \phi_{y}(x, y)\right) \mathrm{d} x \mathrm{~d} y \\
& -\int_{0}^{l}\left\langle\psi_{b v}(y), L(y) \phi_{b v}(y)\right\rangle_{\mathbb{C}^{4}} \mathrm{~d} y,
\end{aligned}
$$

as $Q_{P, L}^{(2)}[\psi]:=Q_{P, L}^{(2)}[\psi, \psi]$. For simplicity we use the same symbol for both forms as it will be clear from the context which form is meant.

Theorem 3.1. Given maps $P, L:[0, l] \rightarrow \mathrm{M}(4, \mathbb{C})$ as above that are bounded and measurable, the quadratic form $Q_{P, L}^{(2)}[\cdot]$ with domain

$$
\mathcal{D}_{Q^{(2)}}=\left\{\psi \in H^{1}(D) ; P(y) \psi_{b v}(y)=0 \text { for a.e. } y \in[0, l]\right\}
$$

is closed and semi-bounded.

Proof. As $L(y)$ is self-adjoint, the expression (3.4) obviously defines a quadratic form on the domain (3.5) in $L^{2}(D)$. We then observe that

$$
\left|\int_{0}^{l}\left\langle\psi_{b v}(y), L(y) \psi_{b v}(y)\right\rangle_{\mathbb{C}^{4}} \mathrm{~d} y\right| \leq L_{\max }\left\|\psi_{b v}\right\|_{L^{2}(0, l) \otimes \mathbb{C}^{4}}^{2},
$$

where

$$
L_{\max }:=\sup _{y \in[0, l]}\|L(y)\|_{o p}
$$


Moreover, as a consequence of Lemma 8 in [Kuc04,

$$
\left\|\psi_{b v}\right\|_{L^{2}(0, l) \otimes \mathbb{C}^{4}}^{2} \leq 4\left(\frac{2}{\delta}\|\psi\|_{L^{2}(D)}^{2}+\delta\|\nabla \psi\|_{L^{2}(D)}^{2}\right)
$$

holds for any $\delta \leq l$. Therefore,

$$
Q_{P, L}^{(2)}[\psi] \geq\left(1-4 \delta L_{\max }\right)\|\nabla \psi\|_{L^{2}(D)}^{2}-\frac{8 L_{\max }}{\delta}\|\Psi\|_{L^{2}(D)}^{2}
$$

Now choose $\delta \leq \frac{1}{4 L_{\max }}$, then there obviously exits $C>0$ such that

$$
Q_{P, L}^{(2)}[\psi] \geq-C\|\psi\|_{L^{2}(D)}^{2}
$$

and hence the quadratic form is bounded from below. We denote the optimal such constant by $C_{\infty}$.

In order to show that the quadratic form (3.4) is closed we observe that the (squared) form norm

$$
\|\cdot\|_{Q_{P, L}^{(2)}}^{2}=Q_{P, L}^{(2)}[\cdot]+\left(C_{\infty}+1\right)\|\cdot\|_{L^{2}(D)}^{2}
$$

is equivalent to the Sobolev norm in $H^{1}(D)$. This follows from (3.8). Therefore, due to the completeness of $H^{1}(D)$ any Cauchy sequence $\left\{\psi_{n}\right\}_{n \in \mathbf{N}}$ in $\mathcal{D}_{Q^{(2)}} \subset H^{1}(D)$ with respect to the form-norm has a limit $\psi \in H^{1}(D)$. In order to see that this limit is also in $\mathcal{D}_{Q^{(2)}}$ we recall that according to the trace theorem (see [Neč67, Dob05] where Lipschitz domains are covered) there exists a constant $c>0$ (depending on the domain $D$ ), such that

$$
\|\gamma \phi\|_{L^{2}(\partial D)}<c\|\phi\|_{H^{1}(D)}
$$

for all $\phi \in H^{1}(D)$, where $\gamma: H^{1}(D) \rightarrow L^{2}(\partial D)$ is the trace map, assigning boundary values on $\partial D$ to functions on $D$. In our notation, this trace map is effectively given by the expression on the left in (3.2), and we will therefore use $\phi_{b v}$ and $\gamma \phi$ interchangeably. (We remark that in our context the estimate (3.12) immediately follows from (3.8).) Thus, $\left\{\psi_{n, b v}\right\}$ converges to $\psi_{b v}$ in $L^{2}(0, l) \otimes \mathbb{C}^{4}$. As the operator $\Pi$ on $L^{2}(0, l) \otimes \mathbb{C}^{4}$ is supposed to be bounded, one concludes that $P(\cdot) \psi_{n ; b v}=0$ converges to $P(\cdot) \psi_{b v}$ and hence $P(y) \psi_{b v}(y)=0$ for a.e. $y \in[0, l]$.

We shall now identify the self-adjoint operator $H$ with domain $\mathcal{D}(H) \subset \mathcal{D}_{Q^{(2)}}$ that derives from this quadratic form according to the representation theorem for quadratic forms (see, e.g., Kat66]). In order to specify $H$ and its domain we use that for all $\phi \in \mathcal{D}(H)$ there exists a unique $\chi \in L^{2}(D)$, depending on $\phi$, such that for all $\psi \in \mathcal{D}_{Q^{(2)}}$ the sesquilinear form is

$$
Q_{P, L}^{(2)}[\phi, \psi]=\langle\chi, \psi\rangle_{L^{2}(D)}
$$

We then need to find $H$ and $\mathcal{D}(H)$ such that $\chi=H \phi$ for all $\phi \in \mathcal{D}(H)$. 
Our first approach is based on the identification of (3.13) as an abstract boundary value problem, following [Sho77]. To this end we split the sesquilinear form as

$$
Q_{P, L}^{(2)}[\phi, \psi]=q_{1}[\phi, \psi]+q_{2}[\phi, \psi]
$$

where $q_{1}$ and $q_{2}$ are, in an obvious way, given by the two terms in (3.4). The second part is a boundary contribution and, strictly speaking, involves the linear, continuous trace map $\gamma: H^{1}(D) \rightarrow L^{2}(\partial D)$.

The abstract boundary value problem requires an abstract Green's operator $\partial_{n}$ that is constructed as follows (see, e.g., [Sho77]): The trace map, restricted to the Hilbert space $\mathcal{D}_{Q^{(2)}}$ (equipped with the form-norm), has kernel ker $\gamma=\mathcal{D}_{Q^{(2)}} \cap H_{0}^{1}(D)$. Let

$$
\mathcal{D}_{0}:=\left\{\psi \in \mathcal{D}_{Q^{(2)}} ; \Delta_{2} \psi \in L^{2}(D)\right\}
$$

then $\partial_{n}: \mathcal{D}_{0} \rightarrow(\operatorname{ran} \gamma)^{\prime}$ is a linear map defined by the relation

$$
q_{1}[\psi, \phi]-\left\langle-\Delta_{2} \psi, \phi\right\rangle_{L^{2}(D)}=\partial_{n} \psi[\gamma \phi], \quad \phi \in \mathcal{D}_{Q^{(2)}}
$$

Notice that whenever $\psi \in H^{2}(D)$ we have

$$
\partial_{n} \psi[\gamma \phi]=\int_{\partial D} \frac{\partial \bar{\psi}}{\partial n} \phi \mathrm{d} s,
$$

so that the operator $\partial_{n}$ is the standard normal derivative and (3.16) is the classical first Green's theorem. In the general case $\partial_{n}$ can be seen as a weak form of a normal derivative, thus justifying our notation.

We are now in a position to apply Theorem 3.A from Sho77] to (3.13) which yields the following result.

Proposition 3.2. Let $H$ be the unique self-adjoint, semi-bounded operator corresponding to the quadratic form $Q_{P, L}^{(2)}$. Then its domain is given by

$$
\mathcal{D}(H)=\left\{\psi \in \mathcal{D}_{0} ; \partial_{n} \psi[\gamma \phi]+q_{2}[\psi, \phi]=0, \forall \phi \in \mathcal{D}_{Q^{(2)}}\right\} .
$$

Due to the presence of the abstract Green's operator this characterisation of the domain is not very explicit. Our aim is to show that, in certain cases, the domain (3.18) coincides with (3.3). The domains $\mathcal{D}_{2}(P, L)$, however, are subspaces of $H^{2}(D)$, a property that does not immediately follow from (3.18). In the theory of partial differential equations this question is well known as the problem of elliptic regularity (see, e.g., GT83]).

Definition 3.3. The quadratic form $Q_{P, L}^{(2)}$ is called regular, iff its associated self-adjoint operator $H$ has a domain $\mathcal{D}(H) \subset H^{2}(D)$.

As the form (3.4) involves a boundary integral, in addition to regularity we have to impose a (somewhat mild) condition on the projectors $P$ ensuring that the kernel of the operator $\Pi$ is under sufficient control. 
Lemma 3.4. Let $P:(0, l) \rightarrow \mathrm{M}(4, \mathbb{C})$ be such that its matrix entries are in $C^{1}(0, l)$, then $\operatorname{ran}\left(\left.\gamma\right|_{\mathcal{D}_{Q^{(2)}}}\right)$ is dense in $\operatorname{ker} \Pi$ with respect to the norm of $L^{2}(0, l) \otimes \mathbb{C}^{4}$.

Proof. As $C_{0}^{\infty}(0, l) \otimes \mathbb{C}^{4} \subset L^{2}(0, l) \otimes \mathbb{C}^{4}$ is dense, whenever $\chi \in \operatorname{ker} \Pi \subset L^{2}(0, l) \otimes \mathbb{C}^{4}$ there exists a sequence $\left\{\chi_{n}\right\} \subset C_{0}^{\infty}(0, l) \otimes \mathbb{C}^{4}$ that converges to $\chi$. Moreover, any $\chi_{n} \in$ $C_{0}^{\infty}(0, l) \otimes \mathbb{C}^{4}$ can be extended to some $\psi_{n} \in H^{1}(D)$, such that $\chi_{n}=\psi_{n, b v}$.

Using the orthogonal complement $\Pi^{\perp}$ to the projector $\Pi$ we note that, by the assumption in the lemma, $\Pi^{\perp} \chi_{n} \in C_{0}^{1}(0, l) \otimes \mathbb{C}^{4}$. Again, $\Pi^{\perp} \chi_{n}$ can be extended to a function $\phi_{n} \in H^{1}(D)$, such that $\Pi^{\perp} \chi_{n}=\phi_{n, b v}$. By construction, $P(y) \phi_{n, b v}(y)=0$ so that indeed $\phi_{n} \in \mathcal{D}_{Q^{(2)}}$. Therefore, identifying $\phi_{n, b v}$ with $\gamma \phi_{n}$ we conclude that $\Pi^{\perp} \chi_{n} \in \operatorname{ran}\left(\left.\gamma\right|_{\mathcal{D}_{Q^{(2)}}}\right)$.

Moreover, as $\Pi$ is assumed to be bounded in operator norm there exits $K>0$ such that

$$
\left\|\Pi^{\perp} \chi_{n}-\chi\right\|_{L^{2}(0, l) \otimes \mathbb{C}^{4}}=\left\|\Pi^{\perp}\left(\chi_{n}-\chi\right)\right\|_{L^{2}(0, l) \otimes \mathbb{C}^{4}} \leq K\left\|\chi_{n}-\chi\right\|_{L^{2}(0, l) \otimes \mathbb{C}^{4}} \rightarrow 0,
$$

as $n \rightarrow \infty$. Thus, $\operatorname{ran}\left(\left.\gamma\right|_{\mathcal{D}_{Q^{(2)}}}\right)$ is dense in ker $\Pi$.

In the regular case, and when the matrix entries of $P$ are of class $C^{1}$, we can specify the domain of the operator $H$ more explicitly.

Theorem 3.5. Suppose that the matrix entries of $P:(0, l) \rightarrow \mathrm{M}(4, \mathbb{C})$ are in $C^{1}(0, l)$ and that the quadratic form $Q_{P, L}^{(2)}$ is regular. Then the unique self-adjoint, semi-bounded operator $H$ that is associated with this form is the two-particle Laplacian $-\Delta_{2}$ with domain $\mathcal{D}_{2}(P, L)$.

Proof. Since in the regular case any $\psi \in \mathcal{D}(H)$ is in $H^{2}(D)$, Green's operator $\partial_{n}$ is the standard normal derivative, see (3.17). This would allow to state the 'boundary condition' contained in (3.18) immediately in an explicit way.

Following the one-particle case developed in [Kuc04, however, we shall now proceed in a more direct way as this will confirm the operator $H$ too. For this we choose $\psi$ in (3.13) to be smooth and compactly supported in $D$, vanishing in neighbourhoods of $\partial D$ such that $\psi_{b v}(y)=0$ for all $y \in[0, l]$. Thus

$$
\langle\chi, \psi\rangle_{L^{2}(D)}=\int_{0}^{l} \int_{0}^{l}\left(\bar{\phi}_{x}(x, y) \psi_{x}(x, y)+\bar{\phi}_{y}(x, y) \psi_{y}(x, y)\right) \mathrm{d} x \mathrm{~d} y .
$$

An integration by parts then yields

$$
\langle\chi, \psi\rangle_{L^{2}(D)}=\int_{0}^{l} \int_{0}^{l}\left(-\bar{\phi}_{x x}(x, y)-\bar{\phi}_{y y}(x, y)\right) \psi(x, y) \mathrm{d} x \mathrm{~d} y,
$$

so that $\chi=H \phi=-\Delta_{2} \phi$. Hence the operator $H$ acts as a two-particle Laplacian, and every $\phi \in \mathcal{D}(H)$ must be in $\mathcal{D}\left(-\Delta_{2,0}^{*}\right)$. Now, we choose $\psi \in \mathcal{D}_{Q^{(2)}}$ that is non-zero in a neighbourhood of $\partial D$. Then, in addition to the right-hand side of (3.21), an integration by parts yields the term

$$
-\int_{0}^{l}\left\langle\phi_{b v}^{\prime}(y)+L(y) \phi_{b v}(y), \psi_{b v}(y)\right\rangle_{\mathbb{C}^{4}} \mathrm{~d} y=-\left\langle\phi_{b v}^{\prime}+L \phi_{b v}, \psi_{b v}\right\rangle_{L^{2}(0, l) \otimes \mathbb{C}^{4}},
$$


which must vanish. Since $L(\cdot)$ is self-adjoint, one can rewrite this term as

$$
\int_{\partial D} \frac{\partial \bar{\phi}}{\partial n} \psi \mathrm{d} s+q_{2}[\phi, \psi]
$$

hence its vanishing is precisely a more explicit version of the boundary condition in (3.18).

Furthermore, the condition $P(y) \psi_{b v}(y)=0$ fulfilled by $\psi \in \mathcal{D}_{Q^{(2)}}$ for a.e. $y \in[0, l]$ implies that $\psi_{b v}$ is in the kernel of the orthogonal projector $\Pi$ on $L^{2}(0, l) \otimes \mathbb{C}^{4}$. Hence, the vanishing of (3.22) for all $\psi \in \mathcal{D}_{Q^{(2)}}$, together with the fact that by Lemma 3.4 $\operatorname{ran}\left(\left.\gamma\right|_{\mathcal{D}_{Q^{(2)}}}\right) \subset \operatorname{ker} \Pi$ is dense, implies that $\phi_{b v}^{\prime}+L(\cdot) \phi_{b v}$ is in the kernel of $\Pi^{\perp}$, or

$$
Q(y) \phi_{b v}^{\prime}(y)+Q(y) L(y) \phi_{b v}(y)=0 \text {. }
$$

Furthermore, as $L(y)$ is an endomorphism of $\operatorname{ran} Q(y) \subseteq \mathbb{C}^{4}$, a comparison with (3.3) shows that $\mathcal{D}(H)=\mathcal{D}_{2}(P, L)$.

Remark 3.6. In the general, not necessarily regular, case the integration by parts leading to (3.21) is not possible and the boundary term (3.22) cannot be expressed in the same form as an integral. Nevertheless, weak derivatives and the abstract version of Green's operator allow to interpret the abstract boundary condition $\partial_{n} \psi[\gamma \phi]+q_{2}[\psi, \phi]=0$ as a distributional variant of (3.24). Regardless of regularity, we shall therefore also use $\mathcal{D}_{2}(P, L)$ to denote the domain of the self-adjoint operator $H$ associated with the quadratic form $Q_{P, L}^{(2)}$.

So far we did not consider to what extent the representation of the quadratic form in terms of the maps $P$ and $L$, see (3.4) and (3.5), is unique.

Proposition 3.7. Suppose that the matrix entries of $P:(0, l) \rightarrow \mathrm{M}(4, \mathbb{C})$ are in $C^{1}(0, l)$. Then the parametrisation of the quadratic form $Q_{P, L}^{(2)}$ in terms of $P$ and $L$ according to (3.4) and (3.5) is unique with this property.

Proof. The characterisation (3.5) of a domain $\mathcal{D}_{Q^{(2)}}$ involves only $P$. Suppose that a given domain can be characterised by two different maps $P_{j}:(0, l) \rightarrow \mathrm{M}(4, \mathbb{C}), j=1,2$, both of which with matrix entries in $C^{1}(0, l)$. The associated projection operators $\Pi_{j}$ on $L^{2}(0, l) \otimes \mathbb{C}^{4}$ are, therefore, different implying ker $\Pi_{1} \neq \operatorname{ker} \Pi_{2}$. We can hence assume that there exists $\chi \in \operatorname{ker} \Pi_{1}$ such that $\chi \notin \operatorname{ker} \Pi_{2}$. Now, following Lemma 3.4 there exists a sequence $\left\{\phi_{n}\right\}$ in $\mathcal{D}_{Q^{(2)}}$ such that $\phi_{n, b v}$ converges to $\chi$. Moreover, following our assumption $\phi_{n} \in \mathcal{D}_{Q^{(2)}}$ means that $\phi_{n, b v} \in \operatorname{ker} \Pi_{1} \cap \operatorname{ker} \Pi_{2}$. However, $\chi \notin \operatorname{ker} \Pi_{2}$ contradicts the fact that the $\phi_{n, b v} \in \operatorname{ker} \Pi_{2}$ converge to $\chi$.

Now assume that a domain $\mathcal{D}_{Q^{(2)}}$ (with a unique $C^{1}$-map $P$ ) is given, but the form (3.4) can be characterised by two different maps $L_{j}:(0, l) \rightarrow \mathrm{M}(4, \mathbb{C}), j=1,2$, yielding two different (bounded and self-adjoint) operators $\Lambda_{j}$ on $L^{2}(0, l) \otimes \mathbb{C}^{4}$. Hence

$$
\left\langle\phi_{b v},\left(\Lambda_{1}-\Lambda_{2}\right) \phi_{b v}\right\rangle_{L^{2}(0, l) \otimes \mathbb{C}^{4}}=0, \quad \text { for all } \phi \in \mathcal{D}_{Q^{(2)}} .
$$

Again following Lemma 3.4, and using that, by definition, $L_{j}(y)$ vanishes on $(\operatorname{ker} P(y))^{\perp}$, this implies $\Lambda_{1}=\Lambda_{2}$. 
Remark 3.8. In the regular case, when the associated operators are two-particle Laplacians with domains $\mathcal{D}_{2}(P, L)$, the same uniqueness results holds for the operators, as the association between closed, semi-bounded quadratic forms and semi-bounded, self-adjoint operators is one-to-one [Kat66].

In a couple of standard cases it is well known that the quadratic form (3.4) is regular, including the forms associated with the following operators:

1. A Dirichlet-Laplacian, in which case $P(y)=\mathbb{1}_{4}$ for all $y \in[0, l]$.

2. A Neumann-Laplacian, where $P(y)=0=L(y)$ for all $y \in[0, l]$.

3. A mixed Dirichlet-Neumann Laplacian, where $P(y)$ is independent of $y$ and diagonal such the diagonal entries are either zero or one. Moreover, $L(y)=0$ for all $y \in[0, l]$. In such a case Dirichlet boundary conditions are imposed on the parts of the boundary that, via (3.2), correspond to a one on the diagonal of $P$, and Neumann boundary conditions on the remaining parts.

4. A Laplacian with standard Robin boundary condition follows when $P(y)=0$ for all $y \in[0, l]$ and $L=\alpha \mathbb{1}_{4}$, where $\alpha>0$. In that case the boundary conditions in (3.3) reduce to $\psi_{b v}^{\prime}(y)+\alpha \psi_{b v}(y)=0$.

We are now going to establish regularity in a further class of examples. Suppose that $P$ takes the following block-diagonal form,

$$
P(y)=\left(\begin{array}{cc}
\tilde{P}(y) & 0 \\
0 & \tilde{P}(y)
\end{array}\right) .
$$

This structure will be necessary in the case of identical particles, see Section 4. We also assume that the matrix entries of $\tilde{P}$ are in $C^{3}(0, l)$ and possess extensions of class $C^{3}$ to some interval $(-\eta, l+\eta), \eta>0$. Then the rank of $\tilde{P}(y)$, which is either zero, one or two, is the same for all $y \in[0, l]$. In the case $\operatorname{rk} \tilde{P}=0$ the only possible solution for $\tilde{P}$ is to be zero, and for rk $\tilde{P}=2$ the projector has to be $\mathbb{1}_{2}$. These to cases are covered by the examples 1 . and 2. above. When $\operatorname{rk} \tilde{P}=1$, the projector is of the form

$$
\tilde{P}(y)=\left(\begin{array}{cc}
\beta(y) & \bar{\gamma}(y) \\
\gamma(y) & 1-\beta(y)
\end{array}\right)
$$

where $0 \leq \beta(y) \leq 1$ and $|\gamma(y)|^{2}=\beta(y)-\beta^{2}(y)$, i.e., when $\gamma(y)=0, \beta(y)$ must be either one or zero. Hence, demanding that $\gamma(y) \rightarrow 0$ as $y \rightarrow 0$ and as $y \rightarrow l, \tilde{P}(y)$ approaches one of the two cases

$$
\left(\begin{array}{ll}
1 & 0 \\
0 & 0
\end{array}\right), \quad\left(\begin{array}{ll}
0 & 0 \\
0 & 1
\end{array}\right)
$$

We shall indeed suppose that on small intervals $\left[0, \varepsilon_{1}\right]$ and $\left[l-\varepsilon_{2}, l\right]$, with $\varepsilon_{j}>0$, the projector $\tilde{P}(y)$ assumes this form as well as that $L(y)=0$. This means that the conditions $P(y) \phi_{b v}(y)=0$ and $Q(y) \phi_{b v}^{\prime}(y)$ imply mixed Dirichlet-Neumann conditions in neighbourhoods of two of the corners of $\partial D$ and either Dirichlet or Neumann conditions in neighbourhoods of the remaining two corners. In such cases we are able to prove regularity. 
Theorem 3.9. Let $L$ be Lipschitz continuous on $[0, l]$ and let $P$ be of the block-diagonal form (3.26). Assume that the matrix entries of $\tilde{P}$ are in $C^{3}(0, l)$ and possess extensions of class $C^{3}$ to some interval $(-\eta, l+\eta), \eta>0$. Moreover, when $y \in\left[0, \varepsilon_{1}\right] \cup\left[l-\varepsilon_{2}, l\right]$, with some $\varepsilon_{1}, \epsilon_{2}>0$, suppose that $L(y)=0$ and that $\tilde{P}(y)$ is diagonal with diagonal entries that are either zero or one. Then the quadratic form $Q_{P, L}^{(2)}$ is regular.

The proof is rather technical and we therefore defer it to the appendix.

We shall finally identify the self-adjoint realisations of the two-particle Laplacian that represent actual interactions. For this purpose we have to identify among the self adjoint realisations $\left(-\Delta_{2}, \mathcal{D}_{2}(P, L)\right)$ those that arise as lifts of a one-particle Laplacians to $\mathcal{H}_{2}$. Hence, let $P^{(1)}$ and $L^{(1)}$ be a projector and a self-adjoint operator on $\mathbb{C}^{2}$, defining a domain (2.7) for a one-particle Laplacian on $[0, l]$. The associated quadratic form and its domain is given by (2.8) and (2.9). Its lift to $\mathcal{H}_{2}$ then is the quadratic form (3.4) defined on the domain (3.5), where

$$
\tilde{P}(y)=\left(\begin{array}{cc}
P^{(1)} & 0 \\
0 & P^{(1)}
\end{array}\right) \quad \text { and } \quad \tilde{L}(y)=\left(\begin{array}{cc}
L^{(1)} & 0 \\
0 & L^{(1)}
\end{array}\right)
$$

for all $y \in[0, l]$. Together with Proposition 3.7 this now leads to a complete characterisation of the interacting vs. the non-interacting representations of the two-particle Laplacian.

Proposition 3.10. Suppose that the matrix entries of $P:(0, l) \rightarrow \mathrm{M}(4, \mathbb{C})$ are in $C^{1}(0, l)$. Then the two-particle Laplacian $-\Delta_{2}$ with domain $\mathcal{D}_{2}(P, L)$ represents no interactions, iff $P$ and $L$ are block-diagonal as in (3.29) and are independent of $y$.

We remark that, clearly, a Dirichlet-, a Neumann- or a standard Robinon-Laplacian on $D$ represent no interactions.

\subsection{Two particles on a general compact metric graph}

The construction of self-adjoint realisations of the two-particle Laplacian on a general compact, metric graph will be based on the above results for the interval. It will use the same methods and mainly involves the introduction of a suitable notation.

For convenience we shall again first treat two distinguishable particles, for which the two-particle Hilbert space is

$$
\mathcal{H}_{2}=L^{2}(\Gamma) \otimes L^{2}(\Gamma)=\bigoplus_{e_{1} e_{2}} L^{2}\left(D_{e_{1} e_{2}}\right)
$$

Here $D_{e_{1} e_{2}}=\left(0, l_{e_{1}}\right) \times\left(0, l_{e_{2}}\right)$ denotes the rectangle on which the component $\psi_{e_{1} e_{2}} \in$ $L^{2}\left(D_{e_{1} e_{2}}\right)$ of $\Psi=\left(\psi_{e_{1} e_{2}}\right) \in \mathcal{H}_{2}$ is defined. In this sense one can view the vectors in $\mathcal{H}_{2}$ as functions on the disjoint union

$$
D_{\Gamma}:=\bigcup_{e_{1} e_{2}}^{\cdot} D_{e_{1} e_{2}}
$$


of $E^{2}$ rectangles. With this motivation in mind we shall also denote $\mathcal{H}_{2}$ as $L^{2}\left(D_{\Gamma}\right)$, and other function spaces such as $H^{m}\left(D_{\Gamma}\right)$ accordingly. The trace map $\gamma$ defined on $H^{1}\left(D_{\Gamma}\right)$ then assigns each function its boundary values, i.e., its values on the disjoint union

$$
\partial D_{\Gamma}:=\bigcup_{e_{1} e_{2}}^{\cdot} \partial D_{e_{1} e_{2}}
$$

of the boundaries of the rectangles $D_{e_{1} e_{2}}$.

A two-particle Laplacian $-\Delta_{2,0}$ can be defined on the domain $\mathcal{D}\left(-\Delta_{2,0}\right)=C_{0}^{\infty}\left(D_{\Gamma}\right)$, on which it is a symmetric, non-self adjoint operator. Following (2.16), it acts on a state $\Psi$ in its domain as

$$
\left(-\Delta_{2,0} \Psi\right)_{e_{1} e_{2}}\left(x_{e_{1}}, y_{e_{2}}\right)=-\psi_{e_{1} e_{2}, x x}\left(x_{e_{1}}, y_{e_{2}}\right)-\psi_{e_{1} e_{2}, y y}\left(x_{e_{1}}, y_{e_{2}}\right) .
$$

The domain $\mathcal{D}\left(-\Delta_{2,0}^{*}\right)$ of its adjoint $-\Delta_{2,0}^{*}$ is the immediate analogue of (3.1) .

Simplifying notation we shall sometimes use a rescaling of variables in that we set

$$
\psi_{e_{1} e_{2}}\left(x_{e_{1}}, y_{e_{2}}\right)=\psi_{e_{1} e_{2}}\left(l_{e_{1}} x, l_{e_{2}} y\right)
$$

with $x, y \in(0,1)$. This then requires to modify the $4 E^{2}$ boundary values of functions $\Psi \in H^{1}\left(D_{\Gamma}\right)$ and derivatives of functions $\Psi \in H^{2}\left(D_{\Gamma}\right)$ as compared to (3.2),

$$
\Psi_{b v}(y)=\left(\begin{array}{c}
\sqrt{l_{e_{2}}} \psi_{e_{1} e_{2}}\left(0, l_{e_{2}} y\right) \\
\sqrt{l_{e_{2}}} \psi_{e_{1} e_{2}}\left(l_{e_{1}}, l_{e_{2}} y\right) \\
\sqrt{l_{e_{1}}} \psi_{e_{1} e_{2}}\left(l_{e_{1}} y, 0\right) \\
\sqrt{l_{e_{1}}} \psi_{e_{1} e_{2}}\left(l_{e_{1}} y, l_{e_{2}}\right)
\end{array}\right) \quad \text { and } \quad \Psi_{b v}^{\prime}(y)=\left(\begin{array}{c}
\sqrt{l_{e_{2}}} \psi_{e_{1} e_{2}, x}\left(0, l_{e_{2}} y\right) \\
-\sqrt{l_{e_{2}}} \psi_{e_{1} e_{2}, x}\left(l_{e_{1}}, l_{e_{2}} y\right) \\
\sqrt{l_{e_{1}}} \psi_{e_{1} e_{2}, y}\left(l_{e_{1}} y, 0\right) \\
-\sqrt{l_{e_{1}}} \psi_{e_{1} e_{2}, y}\left(l_{e_{1}} y, l_{e_{2}}\right)
\end{array}\right)
$$

Here $y \in[0,1]$ and the indices $e_{1} e_{2}$ run over all $E^{2}$ possible pairs with $e_{1}, e_{2}=1, \ldots, E$. As in the case of two particles on an interval, $\Psi_{b v} \in L^{2}(0,1) \otimes \mathbb{C}^{4 E^{2}}$ is a convenient way to parametrise the trace $\gamma \Psi \in L^{2}\left(D_{\Gamma}\right)$ of $\Psi \in H^{1}\left(D_{\Gamma}\right)$.

In order to formulate appropriate boundary conditions we introduce maps $P, L:[0,1] \rightarrow$ $\mathrm{M}\left(4 E^{2}, \mathbb{C}\right)$ such that

1. $P(y)$ is an orthogonal projector,

2. $L(y)$ is a self-adjoint endomorphism on $\operatorname{ker} P(y)$,

for a.e. $y \in[0,1]$; moreover $Q(y)=\mathbb{1}_{4 E^{2}}-P(y)$. As previously, these maps are required to be bounded and measurable. Moreover, operators $\Pi$ and $\Lambda$ can be defined on $L^{2}(0,1) \otimes \mathbb{C}^{4 E^{2}}$ through $(\Pi \chi)(y):=P(y) \chi(y)$ and $(\Lambda \chi)(y):=L(y) \chi(y)$, respectively. As $P$ and $L$ are bounded and measurable functions on $[0,1]$, these operators are bounded. Again, $\Pi$ is a projector and $\Lambda$ is self-adjoint.

This notation now allows to define the following domains for two-particle Laplacians,

$$
\begin{aligned}
\mathcal{D}_{2}(P, L):=\{\Psi & \in H^{2}\left(D_{\Gamma}\right) ; P(y) \Psi_{b v}(y)=0 \text { and } \\
& \left.Q(y) \Psi_{b v}^{\prime}(y)+L(y) Q(y) \Psi_{b v}(y)=0 \text { for a.e. } y \in[0,1]\right\}
\end{aligned}
$$


in close analogy to (3.3). A representation of these domains in terms of maps $A, B:[0,1] \rightarrow$ $\mathrm{M}\left(4 E^{2}, \mathbb{C}\right)$ can be provided in the same manner as for two particles on an interval.

In order to address the question of self-adjointness we follow the strategy outlined above and first generate a suitable quadratic form,

$$
\begin{aligned}
Q_{P, L}^{(2)}[\Psi]: & =\langle\nabla \Psi, \nabla \Psi\rangle_{L^{2}\left(D_{\Gamma}\right)}-\left\langle\Psi_{b v}, \Lambda \Psi_{b v}\right\rangle_{L^{2}(0,1) \otimes \mathbb{C}^{4 E^{2}}} \\
= & \sum_{e_{1}, e_{2}=1}^{E} \int_{0}^{l_{e_{2}}} \int_{0}^{l_{e_{1}}}\left(\left|\psi_{e_{1} e_{2}, x}(x, y)\right|^{2}+\left|\psi_{e_{1} e_{2}, y}(x, y)\right|^{2}\right) \mathrm{d} x \mathrm{~d} y \\
& -\int_{0}^{1}\left\langle\Psi_{b v}(y), L(y) \Psi_{b v}(y)\right\rangle_{\mathbb{C}^{4 E^{2}}} \mathrm{~d} y .
\end{aligned}
$$

This allows us to generalise Theorems 3.1 and 3.5 to general compact metric graphs.

Theorem 3.11. Given maps $P, L:[0,1] \rightarrow \mathrm{M}\left(4 E^{2}, \mathbb{C}\right)$ as above that are bounded and measurable, the quadratic form (3.37) with domain

$$
\mathcal{D}_{Q^{(2)}}=\left\{\Psi \in H^{1}\left(D_{\Gamma}\right) ; P(y) \Psi_{b v}(y)=0 \text { for a.e. } y \in[0,1]\right\}
$$

is closed and semi-bounded.

The proof can be taken over verbatim from Theorem 3.1 when the notation is adapted to the slightly more complex situation of a general graph.

The procedure to extract the self-adjoint operator $H$ with domain $\mathcal{D}(H)$ associated with the quadratic form $Q_{P, L}^{(2)}$ will be the same as in Section 3.1. The notation set out above allows us to copy the procedure verbatim, only replacing $D$ by $D_{\Gamma}$. This first leads to an abstract Green's operator associated with the trace map $\gamma: H^{1}\left(D_{\Gamma}\right) \rightarrow L^{2}\left(\partial D_{\Gamma}\right)$, and then to the equivalent of Proposition 3.2 .

Proposition 3.12. Let $H$ be the unique self-adjoint, semi-bounded operator corresponding to the quadratic form $Q_{P, L}^{(2)}$. Then its domain is given by

$$
\mathcal{D}(H)=\left\{\Psi \in \mathcal{D}_{0} ; \partial_{n} \Psi[\gamma \Phi]+q_{2}[\Psi, \Phi]=0, \forall \Phi \in \mathcal{D}_{Q^{(2)}}\right\}
$$

Again, one would like to have a more explicit description of the domain. This is available when $\Psi \in H^{2}\left(D_{\Gamma}\right)$ as then

$$
\partial_{n} \Psi(\gamma \Phi)=\sum_{e_{1} e_{2}} \int_{\partial D_{e_{1} e_{2}}} \frac{\partial \bar{\psi}_{e_{1} e_{2}}}{\partial n} \phi_{e_{1} e_{2}} \mathrm{~d} s
$$

The Definition 3.3 of regularity can be extended in an obvious way, specifying cases in which the domain of the self-adjoint operator can be given more explicitly by using (3.40). As a result, one obtains the following statement, which can be proved in complete analogy to Lemma 3.4 . 
Lemma 3.13. Let $P:(0,1) \rightarrow \mathrm{M}\left(4 E^{2}, \mathbb{C}\right)$ be such that its matrix entries are in $C^{1}(0,1)$, then $\operatorname{ran}\left(\left.\gamma\right|_{\mathcal{D}_{Q^{(2)}}}\right)$ is dense in ker $\Pi$ with respect to the norm of $L^{2}(0,1) \otimes \mathbb{C}^{4 E^{2}}$.

If the condition in the lemma is fulfilled and the quadratic form is regular we obtain an analogue of Theorem 3.5 .

Theorem 3.14. Suppose that the matrix entries of $P:(0,1) \rightarrow \mathrm{M}\left(4 E^{2}, \mathbb{C}\right)$ are in $C^{1}(0,1)$ and that the quadratic form $Q_{P, L}^{(2)}$ is regular. Then the unique self-adjoint, semi-bounded operator $H$ that is associated with this form is the two-particle Laplacian $-\Delta_{2}$ with domain $\mathcal{D}_{2}(P, L)$.

Proof. The proof is in close analogy to the proof of Theorem 3.11, and leads to an obvious generalisation of (3.21). Performing the integration by part with some $\Psi \in \mathcal{D}_{Q^{(2)}}$ that does not vanish in a neighbourhood of $\partial D_{\Gamma}$ one obtains the additional term

$$
-\int_{0}^{1}\left\langle\Phi_{b v}^{\prime}(y)+L(y) \Phi_{b v}(y), \Psi_{b v}(y)\right\rangle_{\mathbb{C}^{4 E^{2}}} \mathrm{~d} y=-\left\langle\Phi_{b v}^{\prime}+L \Phi_{b v}, \Psi_{b v}\right\rangle_{L^{2}(0,1) \otimes \mathbb{C}^{4 E^{2}}}
$$

This is the explicit expression for $\partial_{n} \Psi[\gamma \Phi]+q_{2}[\Psi, \Phi]$ and is required to vanish. Again, the fact that $\operatorname{ran}\left(\left.\gamma\right|_{\mathcal{D}_{Q^{(2)}}}\right)$ is dense in ker $\Pi$ implies the condition

$$
Q(y) \Psi_{b v}^{\prime}(y)+L(y) Q(y) \Psi_{b v}(y)=0 \text { for a.e. } y \in[0,1]
$$

in (3.36).

Uniqueness of the parametrisation of the quadratic form in terms of maps $P$ and $L$, see (3.37) and (3.38) follows in the same way as on an interval, see Proposition 3.7.

Proposition 3.15. Suppose that the matrix entries of $P:(0,1) \rightarrow \mathrm{M}\left(4 E^{2}, \mathbb{C}\right)$ are in $C^{1}(0,1)$. Then the parametrisation of the quadratic form $Q_{P, L}^{(2)}$ in terms of $P$ and $L$ according to (3.37) and (3.38) is unique with this property.

We are now going to establish the regularity of the quadratic form $Q_{P, L}^{(2)}$ in a class of examples that are similar to Theorem 3.9 . We assume that the projectors $P(y)$ possess a block-diagonal form in analogy to (3.26), where the two blocks $\tilde{P}$ correspond to the $2 E^{2}$ dimensional subspaces spanned by the upper and lower half, respectively, of the components in (3.35). We also suppose that the matrix entries of $\tilde{P}$ are in $C^{3}(0,1)$ with an extension of class $C^{3}$ to some interval $(-\eta, 1+\eta), \eta>0$. As in the case of an interval we need to impose either Dirichlet, Neumann or mixed Dirichlet-Neumann boundary conditions near corners of the rectangles $D_{e_{1} e_{2}}$. This will be achieved when $\tilde{P}(y)$ is diagonal and the diagonal entries are either zero or one.

Theorem 3.16. Let $L$ be Lipschitz continuous on $[0,1]$ and let $P$ be of the block-diagonal form (3.26). Assume that the matrix entries of $\tilde{P}$ are in $C^{3}(0,1)$ and possess extensions of class $C^{3}$ to some interval $(-\eta, 1+\eta), \eta>0$. Moreover, when $y \in\left[0, \varepsilon_{1}\right] \cup\left[l-\varepsilon_{2}, l\right]$, with some $\varepsilon_{1}, \varepsilon_{2}>0$, suppose that $L(y)=0$ and that $\tilde{P}(y)$ is diagonal with diagonal entries that are either zero or one. Then the quadratic form $Q_{P, L}^{(2)}$ is regular.

The proof of this result is essentially the same as that of Theorem 3.9, see the appendix, and mainly involves suitable changes in the notation. 


\section{Two identical particles on a graph}

Implementing exchange symmetry for two identical particles requires the bosonic as well as the fermionic two-particle Hilbert spaces $\mathcal{H}_{2, B}$ and $\mathcal{H}_{2, F}$, respectively, as well as a projection of the Hamiltonian operators and their domains to $\mathcal{H}_{2, B / F}$.

\subsection{Bosons and fermions on a general graph}

The Hilbert spaces $\mathcal{H}_{2, B / F}$ consist of states $\Psi=\left(\psi_{e_{1} e_{2}}\right) \in \mathcal{H}_{2}$ that are (anti-) symmetric under a particle exchange. This means that the $E^{2}$ components $\psi_{e_{1} e_{2}} \in L^{2}\left(D_{e_{1} e_{2}}\right)$ satisfy the relations

$$
\psi_{e_{1} e_{2}}\left(x_{e_{1}}, y_{e_{2}}\right)= \pm \psi_{e_{2} e_{1}}\left(y_{e_{2}}, x_{e_{1}}\right)
$$

where the ' + ' corresponds to the bosonic case, and the '-' applies in the fermionic case. Notice that these conditions relate pairs of functions when $e_{1} \neq e_{2}$ and impose conditions on single functions when $e_{1}=e_{2}$. Therefore, only $\frac{1}{2} E(E+1)$ of the $E^{2}$ components of $\Psi$ in $\mathcal{H}_{2, B}$ or $\mathcal{H}_{2, F}$ are independent. In order to keep the notation simpler, however, we shall keep all components in what follows. We shall then use $L^{2}\left(D_{\Gamma}\right)_{s}$ to denote the subspace of symmetric functions (with respect to the particle exchange), and $L^{2}\left(D_{\Gamma}\right)_{a}$ to denote the subspace of anti-symmetric functions i.e., $\mathcal{H}_{2, B}=L^{2}\left(D_{\Gamma}\right)_{s}$ and $\mathcal{H}_{2, F}=L^{2}\left(D_{\Gamma}\right)_{a}$.

When the components are in $H^{m}\left(D_{e_{1} e_{2}}\right), m=1,2$, they fulfil

$$
\psi_{e_{1} e_{2}, x}\left(x_{e_{1}}, y_{e_{2}}\right)= \pm \psi_{e_{2} e_{1}, y}\left(y_{e_{2}}, x_{e_{1}}\right) \quad \text { and } \quad \psi_{e_{1} e_{2}, x x}\left(x_{e_{1}}, y_{e_{2}}\right)= \pm \psi_{e_{2} e_{1}, y y}\left(y_{e_{2}}, x_{e_{1}}\right) \text {. }
$$

We denote the spaces of these functions as $H^{m}\left(D_{\Gamma}\right)_{s}$ and $H^{m}\left(D_{\Gamma}\right)_{s}$, respectively.

In order to realise a particle exchange symmetry of the quadratic forms we have to ensure a particular structure of the maps $P$ and $L$. They have to consist of two identical, diagonal $2 E^{2} \times 2 E^{2}$-blocks,

$$
M(y)=\left(\begin{array}{cc}
\tilde{M}(y) & 0 \\
0 & \tilde{M}(y)
\end{array}\right)
$$

where $M(y)$ either denotes $P(y)$ or $L(y)$, compare also (3.26). Following (3.35), these blocks correspond to the subspaces of boundary values spanned by the upper half and the lower half, respectively, of the components of $\Psi_{b v}$ or $\Psi_{b v}^{\prime}$.

Making use of the rescaling (3.34) and the symmetry relations (4.2) we introduce $2 E^{2}$ component boundary values

$$
\tilde{\Psi}_{b v}(y)=\left(\begin{array}{c}
\sqrt{l_{e_{2}}} \psi_{e_{1} e_{2}}\left(0, l_{e_{2}} y\right) \\
\sqrt{l_{e_{2}}} \psi_{e_{1} e_{2}}\left(l_{e_{1}}, l_{e_{2}} y\right)
\end{array}\right) \quad \text { and } \quad \tilde{\Psi}_{b v}^{\prime}(y)=\left(\begin{array}{c}
\sqrt{l_{e_{2}}} \psi_{e_{1} e_{2}, x}\left(0, l_{e_{2}} y\right) \\
-\sqrt{l_{e_{2}}} \psi_{e_{1} e_{2}, x}\left(l_{e_{1}}, l_{e_{2}} y\right)
\end{array}\right)
$$

of functions $\Psi \in H^{1}\left(D_{\Gamma}\right)_{s / a}$ and derivatives of functions $\Psi \in H^{2}\left(D_{\Gamma}\right)_{s / a}$. In both symmetry classes these boundary values are sufficient to serve as representatives for traces $\gamma \Psi$ of functions $\Psi \in H^{1}\left(D_{\Gamma}\right)_{s / a}$. 
With these notions the quadratic form (3.37) reads

$$
\begin{aligned}
Q_{P, L}^{(2), B / F}[\Psi]= & 2 \sum_{e_{1}, e_{2}=1}^{E} \int_{0}^{l_{e_{2}}} \int_{0}^{l_{e_{1}}}\left|\psi_{e_{1} e_{2}, x}(x, y)\right|^{2} \mathrm{~d} x \mathrm{~d} y \\
& -2 \int_{0}^{1}\left\langle\tilde{\Psi}_{b v}(y), \tilde{L}(y) \tilde{\Psi}_{b v}(y)\right\rangle_{\mathbb{C}^{2 E^{2}}} \mathrm{~d} y
\end{aligned}
$$

and is defined on either of the domains

$$
\mathcal{D}_{Q^{(2), B / F}}=\left\{\Psi \in H^{1}\left(D_{\Gamma}\right)_{s / a} ; \tilde{P}(y) \tilde{\Psi}_{b v}(y)=0 \text { for a.e. } y \in[0,1]\right\} .
$$

Correspondingly, the domain (3.36) converts into

$$
\begin{aligned}
& \mathcal{D}_{2, B / F}(P, L):=\{\Psi \in H^{2}\left(D_{\Gamma}\right)_{s / a} ; \tilde{P}(y) \tilde{\Psi}_{b v}(y)=0 \text { and } \\
&\left.\tilde{Q}(y) \tilde{\Psi}_{b v}^{\prime}(y)+\tilde{L}(y) \tilde{Q}(y) \tilde{\Psi}_{b v}(y)=0 \text { for a.e. } y \in[0,1]\right\} .
\end{aligned}
$$

Theorem 3.11 now carries over immediately to the case of either two bosons or two fermions on the graph. The self-adjoint operator $H_{B / F}$ associated with the quadratic form (4.5) on the domain (4.7) is identified by the immediate analogue of Proposition 3.12 .

Finally, in the case of a regular quadratic form we can again identify the operator and its domain in an explicit way, leading to the main result of this section.

Theorem 4.1. Suppose that the matrix entries of $\tilde{P}:(0,1) \rightarrow \mathrm{M}\left(2 E^{2}, \mathbb{C}\right)$ are in $C^{1}(0,1)$ and that the quadratic form $Q_{P, L}^{(2), B / F}$ is regular. Then the unique self-adjoint, semi-bounded operator $H_{B / F}$ that is associated with this form is the bosonic or fermionic two-particle Laplacian $-\Delta_{2, B / F}$ with domain $\mathcal{D}_{2, B / F}(P, L)$.

Remark 4.2. Examples of regular quadratic forms follow immediately from Theorem 3.16,

We still need to identify those realisations of the (bosonic or fermionic) two-particle Laplacian that represent genuine two-particle interactions. In order to find these we decompose the space of boundary values as

$$
V=\bigoplus_{e_{2}=1}^{E} V_{e_{2}}
$$

where $V_{e_{2}} \cong \mathbb{C}^{2 E}$ is the subspace of partial boundary values $\tilde{\Psi}_{b v, e_{2}}$ and $\tilde{\Psi}_{b v, e_{2}}^{\prime}$ of the components in (4.4) with fixed $e_{2}$. Loosely speaking, $V_{e_{2}}$ contains the boundary values of states for one particle on edge $e_{1}$ under the condition that the second particle is at some point $l_{e_{2}} y$ on egde $e_{2}$.

Proposition 4.3. Suppose that the matrix entries of $\tilde{P}:(0,1) \rightarrow \mathrm{M}\left(2 E^{2}, \mathbb{C}\right)$ are in $C^{1}(0,1)$. Then the two-particle Laplacian $-\Delta_{2, B / F}$ with domain $\mathcal{D}_{2, B / F}(P, L)$ represents no interactions, iff $\tilde{P}$ and $\tilde{L}$ are independent of $y$ and are block-diagonal with respect to the decomposition (4.8), where the blocks are identical and represent corresponding one-particle maps. 
Proof. When $\tilde{P}, \tilde{L}$ are independent of $y$ and are block-diagonal with respect to (4.8), with identical blocks, the domain $\mathcal{D}_{2}(P, L)$ can immediately be split into (identical) one-particle domains. Due to the uniqueness of the representation that follows from Proposition 3.15 every non-interacting two-particle Laplacian must be of this form.

Next we wish to characterise local realisations of the two-particle Laplacian. We recall that for a single particle on a graph locality means that the respective maps $P$ and $L$ are block-diagonal with respect to the decomposition (2.10). For two particles locality has to be considered on two levels: one-particle and two-particle locality. The latter should mean that two particles only interact when they are either on the same edge, or on two edges that are connected in a vertex. On a one-particle level locality means that each of the two particles interacts with the outside only at actual vertices, in the same way as in a one-particle quantum graph.

In more detail, two-particle locality means that boundary conditions should only be imposed on those components of $\Psi=\left(\psi_{e_{1} e_{2}}\right)$ where there is a vertex connecting the edges $e_{1}$ and $e_{2}$. This is implemented by first ordering the components in (4.4) according to the vertices that comprise either the initial end (where the coordinate vanishes), or the final end (where the coordinate takes its maximal value) of edge $e_{1}$. This yields a decomposition of the space of boundary values (4.8) as

$$
V=\bigoplus_{v \in \mathcal{V}} V_{v}
$$

where $V_{v}$ contains all boundary values at edge ends that are connected in the vertex $v$. Two-particles now means that

$$
V_{v}=\bigoplus_{v} V_{l o c a l, v}
$$

Here $V_{\text {local }, v}$ consists of the components $\psi_{e_{1} e_{2}}\left(0, l_{e_{2}} y\right)$ or $\psi_{e_{1} e_{2}}\left(l_{e_{1}}, l_{e_{2}} y\right)$, respectively, where the first variable forms the vertex $v$ and the second variable lives on an edge $e_{2}$ connected to $e_{1}$, either in the vertex $v$ or in the other edge end of $e_{1}$. Locality then requires that the matrices $P(y)$ and $L(y)$ are block diagonal with respect to this decomposition.

\subsection{An Example}

In this section we want to illustrate the meaning of the boundary conditions introduced in the previous paragraphs in a simple example. For this purpose it is sufficient to consider only local properties at a single vertex. We therefore choose the simplest possible example of two identical particles (bosons or fermions) on a graph with one vertex and two half-lines attached to it. Although this graph is not compact and therefore, strictly speaking, is not covered by our results above, these can be carried over in an obvious way.

For convenience we characterise the boundary conditions in terms of the matrix-valued maps $A, B:[0, \infty) \rightarrow \mathrm{M}(4, \mathbb{C})$, see the paragraph below (3.3. $)$. The graph has two edges, 
hence a bosonic or fermionic two-particle state $\Psi=\left(\psi_{e_{1} e_{2}}\right) \in\left(L^{2}\left(\mathbb{R}_{+}^{2}\right) \otimes \mathbb{C}^{4}\right)_{s / a}$ has four components. Boundary values are encoded in

$$
\tilde{\Psi}_{b v}(y)=\left(\begin{array}{l}
\psi_{11}(0, y) \\
\psi_{21}(0, y) \\
\psi_{12}(0, y) \\
\psi_{22}(0, y)
\end{array}\right) \quad \text { and } \quad \tilde{\Psi}_{b v}^{\prime}(y)=\left(\begin{array}{c}
\psi_{11, x}(0, y) \\
\psi_{21, x}(0, y) \\
\psi_{12, x}(0, y) \\
\psi_{22, x}(0, y)
\end{array}\right)
$$

as each edge has only one finite edge end. We then choose the matrices

$$
A(y)=\left(\begin{array}{cccc}
1 & -1 & 0 & 0 \\
0 & v(0, y) & 0 & 0 \\
0 & 0 & 1 & -1 \\
0 & 0 & 0 & v(0,-y)
\end{array}\right) \quad \text { and } \quad B(y)=\left(\begin{array}{cccc}
0 & 0 & 0 & 0 \\
-1 & -1 & 0 & 0 \\
0 & 0 & 0 & 0 \\
0 & 0 & -1 & -1
\end{array}\right)
$$

where $v \in C_{0}^{\infty}\left(\mathbb{R}^{2}\right)$ with $v(x, y)=v(y, x)$.

The boundary conditions $A(y) \tilde{\Psi}_{b v}(y)+B(y) \tilde{\Psi}_{b v}^{\prime}(y)=0$ imply that $\psi_{11}(0, y)=\psi_{21}(0, y)$ and $\psi_{12}(0, y)=\psi_{22}(0, y)$. These conditions ensure continuity of the functions across the vertex in the first variable. Due to the particle exchange symmetry this carries over to the other variable. Moreover,

$$
\begin{aligned}
& -\psi_{11, x}(0, y)-\psi_{21, x}(0, y)=-v(0, y) \psi_{21}(0, y) \\
& -\psi_{12, x}(0, y)-\psi_{22, x}(0, y)=-v(0,-y) \psi_{22}(0, y)
\end{aligned}
$$

We now arrange the four functions $\psi_{e_{1} e_{2}}$ on $\mathbb{R}_{+}^{2}$ into a single function $\psi$ on $\mathbb{R}^{2}$ by defining, for $x, y>0$,

$$
\begin{aligned}
\psi(x, y) & :=\psi_{11}(x, y) \\
\psi(-x,-y) & :=\psi_{22}(x, y) \\
\psi(x,-y) & :=\psi_{12}(x, y) \\
\psi(-x, y) & :=\psi_{21}(x, y)
\end{aligned}
$$

Converting the boundary conditions (4.12) into equivalent conditions for the function $\psi$ then yields a domain for the (formal) Hamiltonian

$$
\hat{H}=-\frac{\partial^{2}}{\partial x^{2}}-\frac{\partial^{2}}{\partial y^{2}}+v(x, y)[\delta(x)+\delta(y)]
$$

for two bosons or fermions on the real line.

This example hence illustrates that the two-particle Laplacians introduced above represent singular two-particle interactions that act when (at least) one particle hits a vertex. 


\section{$5 \quad$ Spectral properties}

We shall now show that the operators constructed in the previous section possess purely discrete spectra and that their eigenvalues are distributed according to an appropriate Weyl law. These observations are certainly not surprising as the operators are composed of Laplacian on a finite number of rectangles. Still, proofs of these statements seem desirable.

If a two-particle Laplacian $-\Delta_{2}$ is a lift of a one-particle Laplacian $-\Delta_{1}$, i.e., $-\Delta_{2}=$ $\left(-\Delta_{1}\right) \otimes \mathbb{1}_{\mathcal{H}_{1}}+\mathbb{1}_{\mathcal{H}_{1}} \otimes\left(-\Delta_{1}\right)$ on the domain $\mathcal{D}_{1}(P, L) \otimes \mathcal{D}_{1}(P, L)$, Theorem VIII.33 in [RS72] implies for the spectrum of the two-particle operator that

$$
\sigma\left(-\Delta_{2}\right)=\sigma\left(-\Delta_{1}\right)+\sigma\left(-\Delta_{1}\right)
$$

in particular, $\sigma\left(-\Delta_{2}\right)$ is purely discrete. (Notice that here $-\Delta_{2}$ is defined on the Hilbert space $\mathcal{H}_{2}$ for two distinguishable particles. A projection to the bosonic or fermionic subspace is yet to follow.) The eigenvalues of $-\Delta_{2}$ are therefore of the form $\lambda_{n, m}=k_{n}^{2}+k_{m}^{2}$, when $\sigma\left(-\Delta_{1}\right)=\left\{k_{n}^{2} ; n \in \mathbb{N}_{0}\right\}$. One can, therefore, immediately determine the eigenvalue asymptotics for such a two-particle Laplacian.

Lemma 5.1. Let $-\Delta_{2}=\left(-\Delta_{1}\right) \otimes \mathbb{1}_{\mathcal{H}_{1}}+\mathbb{1}_{\mathcal{H}_{1}} \otimes\left(-\Delta_{1}\right)$ be a lift of a one-particle Laplacian $\left(-\Delta_{1}, \mathcal{D}(P, L)\right)$ to the two-particle Hilbert space $\mathcal{H}_{2}$. Then the eigenvalues $\left\{\lambda_{n, m} ; n, m \in\right.$ $\left.\mathbb{N}_{0}\right\}$ of $-\Delta_{2}$ are distributed according to the Weyl law,

$$
N_{2}(\lambda):=\left\{(n, m) \in \mathbb{N}_{0}^{2} ; \lambda_{n, m} \leq \lambda\right\} \sim \frac{\mathcal{L}^{2}}{4 \pi} \lambda, \quad \lambda \rightarrow \infty
$$

where $\mathcal{L}=l_{1}+\cdots+l_{E}$ is the sum of the edge lengths of the graph. It is understood that $\mathrm{N}_{2}(\lambda)$ counts eigenvalues with their respective multiplicities.

Proof. It is known [BE09] that for any self-adjoint realisation of the one-particle Laplacian on a compact, metric graph with eigenvalues $k_{n}^{2}$ the eigenvalue count follows a Weyl law,

$$
N_{1}(k):=\left\{n \in \mathbb{N}_{0} ; k_{n}^{2} \leq k^{2}\right\} \sim \frac{\mathcal{L}}{\pi} k, \quad k \rightarrow \infty .
$$

Via a Tauberian theorem Kar31, this asymptotic law is equivalent to

$$
\sum_{n} \mathrm{e}^{-k_{n}^{2} t} \sim \frac{\mathcal{L}}{\sqrt{4 \pi t}}, \quad t \rightarrow 0+.
$$

Squaring both sides of (5.4) and using the equivalence between eigenvalue asymptotics and heat-trace asymptotics in the opposite direction immediately yields (5.2).

Implementing particle exchange symmetry, one observes that the eigenfunctions $\psi_{n} \otimes$ $\psi_{m} \in \mathcal{D}_{1}(P, L) \otimes \mathcal{D}_{1}(P, L)$ and $\psi_{m} \otimes \psi_{n}$ of $-\Delta_{2}$ are transformed into their (anti-) symmetric versions $\psi_{n} \otimes \psi_{m} \pm \psi_{m} \otimes \psi_{n}$. The multiplicity of the eigenvalue $\lambda_{n, m}=\lambda_{m, n}$ is, therefore, 
reduced by a factor of two. Hence, in the bosonic or fermionic case the Weyl asymptotics read

$$
N_{2, B / F}(\lambda) \sim \frac{\mathcal{L}^{2}}{8 \pi} \lambda, \quad \lambda \rightarrow \infty
$$

We shall now generalise the above statements to all realisations (irrespective of regularity) of the two-particle Laplacian introduced in the previous section.

Theorem 5.2. A self-adjoint, bosonic or fermionic realisation of the two-particle Laplacian $-\Delta_{2}$ on a domain $\mathcal{D}_{2, B / F}(P, L)$ has compact resolvent and, therefore, possesses a purely discrete spectrum. Moreover, the eigenvalue asymptotic follow the Weyl law (5.5).

Proof. The proof is based on a comparison with two simple operators (quadratic forms) in the spirit of the well-known Dirichlet-Neumann bracketing. Both comparison operators are lifts of one-particle operators.

The first operator, $\left(-\Delta_{2}, \mathcal{D}_{2, B / F}\left(P_{D}, L_{D}\right)\right)$, is a lift of the one-particle Dirichlet-Laplacian, and is characterised by the projector $P_{D}=\mathbb{1}_{V}$ as well as $L_{D}=0$ on the space (4.8) of boundary values. The second operator, $\left(-\Delta_{2}, \mathcal{D}_{2, B / F}\left(P_{R}, L_{R}\right)\right)$, is a lift of a one-particle Robin-Laplacian, and is characterised by the projector $P_{R}=0$ and $L_{R}=\lambda \mathbb{1}_{V}$, where $\lambda$ is the operator norm of the bounded map $\Lambda$ defined by $L$ (see also [BE09]).

When $\left(-\Delta_{2}, \mathcal{D}_{2, B / F}(P, L)\right)$ is any of the self-adjoint realisations introduced previously, the associated quadratic forms obviously satisfy the following inclusions of their domains,

$$
\mathcal{D}_{Q_{P_{D}, L D}^{(2), B / F}} \subseteq \mathcal{D}_{Q_{P, L}^{(2), B / F}} \subseteq \mathcal{D}_{Q_{P_{R}, L R}^{(2), B / F}}
$$

This means, in the sense of [RS78], that

$$
\left(-\Delta_{2}, \mathcal{D}_{2, B / F}\left(P_{D}, L_{D}\right)\right) \geq\left(-\Delta_{2}, \mathcal{D}_{2, B / F}(P, L)\right) \geq\left(-\Delta_{2}, \mathcal{D}_{2, B / F}\left(P_{R}, L_{R}\right)\right) .
$$

This 'bracketing' is in close analogy to the Dirichlet-Neumann bracketing (see, e.g., [RS78]): Let $H$ be a self-adjoint, semi-bounded operator on a Hilbert space $\mathcal{H}$, and define

$$
\mu_{n}(H):=\sup _{\varphi_{1}, \ldots, \varphi_{n-1} \in \mathcal{H}} \inf _{\substack{\psi \in\left[\varphi_{1}, \ldots, \varphi_{n-1}\right] \perp \\ \psi \in Q_{H},\|\psi\|=1}}\langle\psi, H \psi\rangle_{\mathcal{H}} .
$$

Then, (5.7) implies that

$$
\mu_{n}\left(-\Delta_{2}\right)_{D} \geq \mu_{n}\left(-\Delta_{2}\right) \geq \mu_{n}\left(-\Delta_{2}\right)_{R}
$$

Using that both the Dirichlet- and the Robin-Laplacian have compact resolvent one concludes (with Theorem XIII.64 in [RS78]) that $\mu_{n}\left(-\Delta_{2}\right)_{R, D} \rightarrow \infty$ as $n \rightarrow \infty$; hence the same is true for $\mu_{n}\left(-\Delta_{2}\right)$. By the same theorem this implies that $\left(-\Delta_{2}, \mathcal{D}_{2, B / F}(P, L)\right)$ has compact resolvent.

Furthermore, (5.7) implies for the eigenvalue counting functions that

$$
N_{2, B / F}^{D}(\lambda) \leq N_{2, B / F}(\lambda) \leq N_{2, B / F}^{R}(\lambda) .
$$

As both $N_{2, B / F}^{D}$ and $N_{2, B / F}^{R}$ count eigenvalues of a two-particle Laplacian that is a lift of a one-particle Laplacian they both satisfy the Weyl asymptotics (5.5). Hence the same asymptotics hold for $N_{2, B / F}$. 


\section{Acknowledgement}

J K would like to thank the Evangelisches Studienwerk Villigst for financial support through a Promotionsstipendium.

\section{A A regularity theorem}

In this appendix we prove Theorem 3.9 in Section 4 . We recall that given a quadratic form as in Theorem 3.1 or 3.11, our goal is to establish regularity of the associated selfadjoint operator $H$ with domain (3.18) or (3.39), respectively. To achieve this we need to show that any function $\phi \in \mathcal{D}(H)$ has $H^{2}$-regularity. As the domains $D_{e_{1} e_{2}}$ on which the functions are defined are rectangles, their boundaries have only Lipschitz-regularity and are not smooth. Our approach to this problem utilises an effective cut-off of the corners in combination with the standard difference quotient technique to establish regularity (see, e.g., GT83]). Difference quotients are defined in an obvious way: With $h>0$ and $\phi \in$ $L^{2}(D)$, the difference quotients in the positive and negative direction of the $i$-th coordinate, respectively, are

$$
\begin{aligned}
D_{i}^{+h} \phi(x) & :=\frac{1}{h}\left(\phi\left(x+h e_{i}\right)-\phi(x)\right), \\
D_{i}^{-h} \phi(x) & :=\frac{1}{h}\left(\phi(x)-\phi\left(x-h e_{i}\right)\right),
\end{aligned}
$$

where $e_{i}$ is the corresponding unit vector.

When $\phi \in C^{1}(D)$, limits of difference quotients as $h \rightarrow 0$ clearly yield the corresponding directional derivatives. When a function has weaker regularity estimates of difference quotients allow to conclude weak differentiability, see [Dob05].

Lemma A.1. Let $\Omega$ a bounded domain. If $\phi \in L^{2}(\Omega)$ and $\left\|D_{i}^{+h} \phi\right\|_{L^{2}\left(\Omega_{0}\right)} \leq K$, uniformly for all compact domains $\Omega_{0} \Subset \Omega$ and for all $0<h \leq h_{0}\left(\Omega_{0}\right)$, then $\phi$ is weakly differentiable with respect to $x_{i}$ and $\left\|\phi_{x_{i}}\right\|_{L^{2}(\Omega)} \leq K$.

Another useful result is the following [Dob05].

Lemma A.2. Let $\Omega$ be a bounded domain. Then

$$
\left\|D_{i}^{ \pm h} \phi\right\|_{L^{2}\left(\Omega_{0}^{ \pm}\right)} \leq\left\|\phi_{x_{i}}\right\|_{L^{2}(\Omega)}, \quad \forall \phi \in H^{1}(\Omega),
$$

where $\Omega_{0}^{ \pm} \subset \Omega$ is the maximal domain on which $D_{i}^{ \pm h}(\cdot)$ can be defined. 
Some obvious properties of difference quotients include a 'product rule',

$$
D_{i}^{ \pm h}(\phi \psi)(x)=\left(D_{i}^{ \pm h} \phi\right)(x) \psi(x)+\phi\left(x \pm h e_{i}\right) D_{i}^{ \pm h} \psi(x),
$$

and 'integration by parts'. In one dimension this takes the form

$$
\int_{a}^{b}\left(D_{x}^{+h} \phi(x)\right) \psi(x) \mathrm{d} x=-\int_{a}^{b} \phi(x) D_{x}^{-h} \psi(x) \mathrm{d} x
$$

when the support of either $\psi$ or $\phi$ is contained in $[a-h, b-h]$.

For ease of notation we restrict the following discussion to the case of two particles on an interval; the extension to general graphs will be obvious. In the case of an interval the two-particle configuration space is the rectangle $D$. In order to effectively cut its corners off we choose suitable test functions $\tau \in C^{\infty}(D)$ and show that there exists a constant $K>0$ such that for any $\phi \in \mathcal{D}(H)$ the estimate

$$
\left\|\tau D_{i}^{h} \nabla \phi\right\|_{L^{2}(D)} \leq K
$$

holds for all $h \leq h_{0}$. This then allows to apply Lemma A.1 to eventually conclude that $\phi \in H^{2}(D)$.

For convenience we state here the first result (Theorem 3.9) that we wish to prove in this appendix.

Theorem A.3. Let $L$ be Lipschitz continuous on $[0, l]$ and let $P$ be of the block-diagonal form (3.26). Assume that the matrix entries of $\tilde{P}$ are in $C^{3}(0, l)$ and possess extensions of class $C^{3}$ to some interval $(-\eta, l+\eta), \eta>0$. Moreover, when $y \in\left[0, \varepsilon_{1}\right] \cup\left[l-\varepsilon_{2}, l\right]$, with some $\varepsilon_{1}, \epsilon_{2}>0$, suppose that $L(y)=0$ and that $\tilde{P}(y)$ is diagonal with diagonal entries that are either zero or one. Then the quadratic form $Q_{P, L}^{(2)}$ is regular.

Proof. We first show regularity on any subdomain of the form $D^{\prime}=[0, l] \times[\epsilon, l-\epsilon]$ with $\epsilon>0$, leaving the discussion of regularity in the corners of the domain $D$ until the end. Our first tool is the double difference quotient

$$
\begin{aligned}
D_{y}^{-h} \tau^{2} D_{y}^{+h} \phi(x, y)= & \frac{1}{h^{2}}\left(\tau^{2}(y) \phi(x, y+h)-\tau^{2}(y) \phi(x, y)\right. \\
& \left.-\tau^{2}(y-h) \phi(x, y)+\tau^{2}(y-h) \phi(x, y-h)\right),
\end{aligned}
$$

where $\phi \in \mathcal{D}(H) \subset \mathcal{D}_{Q^{(2)}}$ and $\tau \in C_{0}^{\infty}(\mathbb{R})$ is a test function with support in $(0, l)$ such that $\left.\tau\right|_{[\epsilon, l-\epsilon]}=1$ and $\tau \leq 1$ elsewhere. Even though $\phi$ satisfies the boundary condition $P(y) \phi_{b v}(y)=0$, (A.6) does, in general not. This is due to the dependence of the matrix $P$ on $y$. Therefore, we introduce a correction function $\kappa \in H^{1}(D)$ such that

$$
D_{y}^{-h} \tau^{2} D_{y}^{+h} \phi+\kappa \in \mathcal{D}_{Q^{(2)}} .
$$

We now determine and estimate $\kappa$ and, to this end, insert (A.7) for $\psi$ into (3.13),

$$
\begin{aligned}
\langle\nabla \phi, & \left.\nabla\left(D_{y}^{-h} \tau^{2} D_{y}^{+h} \phi\right)\right\rangle_{L^{2}(D)}+\langle\nabla \phi, \nabla \kappa\rangle_{L^{2}(D)}-\left\langle\phi_{b v}, \Lambda\left(D_{y}^{-h} \tau^{2} D_{y}^{+h} \phi\right)_{b v}\right\rangle_{L^{2}(0, l) \otimes \mathbb{C}^{4}} \\
& -\left\langle\phi_{b v}, \Lambda \kappa_{b v}\right\rangle_{L^{2}(0, l) \otimes \mathbb{C}^{4}}=\left\langle\chi, D_{y}^{-h} \tau^{2} D_{y}^{+h} \phi\right\rangle_{L^{2}(D)}+\langle\chi, \kappa\rangle_{L^{2}(D)} .
\end{aligned}
$$


Employing an integration by parts (A.4), while taking into account that $\tau$ is compactly supported in $y$ and choosing $h$ to be sufficiently small, the first term of (A.8) can be re-written as

$$
\begin{aligned}
\int_{0}^{l} \int_{0}^{l} \nabla \bar{\phi} \nabla\left(D_{y}^{-h} \tau^{2} D_{y}^{+h} \phi\right) \mathrm{d} x \mathrm{~d} y= & -\int_{0}^{l} \int_{0}^{l} \tau^{2}\left|D_{y}^{+h} \nabla \phi\right|^{2} \mathrm{~d} x \mathrm{~d} y \\
& -\int_{0}^{l} \int_{0}^{l}\left(\nabla D_{y}^{+h} \bar{\phi}\right)\left(\partial_{y} \tau^{2}\right)\left(D_{y}^{+h} \phi\right) \mathrm{d} x \mathrm{~d} y .
\end{aligned}
$$

Hence, (A.8) yields

$$
\begin{aligned}
\left\|\tau D_{y}^{+h} \nabla \phi\right\|_{L^{2}(D)}^{2}= & -\left\langle\nabla D_{y}^{+h} \phi, \partial_{y}\left(\tau^{2}\right) D_{y}^{+h} \phi\right\rangle_{L^{2}(D)}+\langle\nabla \phi, \nabla \kappa\rangle_{L^{2}(D)} \\
& -\left\langle\phi_{b v}, \Lambda\left(D_{y}^{-h} \tau^{2} D_{y}^{+h} \phi\right)_{b v}\right\rangle_{L^{2}(0, l) \otimes \mathbb{C}^{4}}-\left\langle\phi_{b v}, \Lambda \kappa_{b v}\right\rangle_{L^{2}(0, l) \otimes \mathbb{C}^{4}} \\
& -\left\langle\chi, D_{y}^{-h} \tau^{2} D_{y}^{+h} \phi\right\rangle_{L^{2}(D)}-\langle\chi, \kappa\rangle_{L^{2}(D)}
\end{aligned}
$$

which allows the estimate

$$
\begin{aligned}
\left\|\tau D_{y}^{+h} \nabla \phi\right\|_{L^{2}(D)}^{2} \leq & \left\|\tau D_{y}^{+h} \nabla \phi\right\|_{L^{2}(D)}\left\|2\left(\partial_{y} \tau\right) D_{y}^{+h} \phi\right\|_{L^{2}(D)}+\|\nabla \phi\|_{L^{2}(D)}\|\nabla \kappa\|_{L^{2}(D)} \\
& +\left|\left\langle\phi_{b v}, \Lambda\left(D_{y}^{-h} \tau^{2} D_{y}^{+h} \phi\right)_{b v}\right\rangle_{L^{2}(0, l) \otimes \mathbb{C}^{4}}\right|+\left|\left\langle\phi_{b v}, \Lambda \kappa_{b v}\right\rangle_{L^{2}(0, l) \otimes \mathbb{C}^{4}}\right| \\
& +\|\chi\|_{L^{2}(D)}\left\|D_{y}^{-h} \tau^{2} D_{y}^{+h} \phi\right\|_{L^{2}(D)}+\|\chi\|_{L^{2}(D)}\|\kappa\|_{L^{2}(D)} .
\end{aligned}
$$

We now use (3.12), following from the trace theorem, to conclude that

$$
\left|\left\langle\phi_{b v}, \Lambda \kappa_{b v}\right\rangle_{L^{2}(0, l) \otimes \mathbb{C}^{4}}\right| \leq C\|\phi\|_{H^{1}(D)}\|\kappa\|_{H^{1}(D)},
$$

where the constant $C>0$ incorporates the constant $c$ from (3.12) as well as the norm of the bounded map $\Lambda$. Furthermore, using the Cauchy-inequality

$$
|a b|<\epsilon a^{2}+\frac{b^{2}}{4 \epsilon}, \quad \forall a, b \in \mathbb{R}, \epsilon>0,
$$

in the first, second and fourth term on the right-hand side of (A.11) we arrive at

$$
\begin{aligned}
\left\|\tau D_{y}^{+h} \nabla \phi\right\|_{L^{2}(D)}^{2} \leq & c_{1}\left(\epsilon_{1}\right)+\epsilon_{1}\left\|\tau \nabla D_{y}^{+h} \phi\right\|_{L^{2}(D)}^{2}+c_{2}\left(\epsilon_{2}\right)+\epsilon_{2}\|\nabla \kappa\|_{L^{2}(D)}^{2} \\
& +\left|\left\langle\phi_{b v}, \Lambda\left(D_{y}^{-h} \tau^{2} D_{y}^{+h} \phi\right)_{b v}\right\rangle_{L^{2}(0, l) \otimes \mathbb{C}^{4}}\right| \\
& +c_{3}\left(\epsilon_{3}\right)+\epsilon_{3}\left(\|\kappa\|_{L^{2}(D)}^{2}+\|\nabla \kappa\|_{L^{2}(D)}^{2}\right) \\
& +\|\chi\|_{L^{2}(D)}\left\|D_{y}^{-h} \tau^{2} D_{y}^{+h} \phi\right\|_{L^{2}(D)}+\|\chi\|_{L^{2}(D)}\|\kappa\|_{L^{2}(D)} .
\end{aligned}
$$

Here we kept all terms containing the still unknown function $\kappa$ or difference quotients of $\nabla \phi$ explicitly, as these are the quantities we want to estimate; all other terms are absorbed in the quantities $c_{j}\left(\epsilon_{j}\right)$.

In order to estimate the fourth, the seventh and the last term on the right-hand side of (A.14) we need to determine a suitable function $\kappa$ and, in particular, show that the bounds

$$
\|\kappa\|_{L^{2}(D)} \leq K_{1} \quad \text { and } \quad\|\nabla \kappa\|_{L^{2}(D)}^{2} \leq K_{2}+K_{3}\left\|\tau D_{y}^{+h} \nabla \phi\right\|_{L^{2}(D)}^{2}
$$


hold, where $K_{j}>0$ are some constants not depending on $h$.

In order to characterise $\kappa$, we infer from (A.7) that its boundary values have to be such that

$$
P(y)\left(\left(D_{y}^{-h} \tau^{2} D_{y}^{+h} \phi\right)_{b v}(y)+\kappa_{b v}(y)\right)=0 .
$$

Expanding the double difference quotient and using $P(y) \phi_{b v}(y)=0$, we obtain a condition of which the upper two components read

$$
\tilde{P}(y)\left(\tau^{2}(y) \tilde{\phi}_{b v}(y+h)+\tau^{2}(y-h) \tilde{\phi}_{b v}(y-h)\right)+h^{2} \tilde{P}(y) \tilde{\kappa}_{b v}(y)=0 ;
$$

here we employed the notation $\tilde{\phi}_{b v}(y)=(\phi(0, y), \phi(l, y))^{T}$ (i.e., a non-rescaled analogue of (4.4)) as well as the block-structure (4.3) of $P$. Since $\tilde{P}(y)$ is a projector, this condition is solved by

$$
\tilde{\kappa}_{b v}(y)=-\frac{1}{h^{2}} \tilde{P}(y)\left(\tau^{2}(y) \tilde{\phi}_{b v}(y+h)+\tau^{2}(y-h) \tilde{\phi}_{b v}(y-h)\right) .
$$

This, however, only yields the boundary values of the function we wish to find. Moreover, the negative power of $h$ would inhibit the envisaged bound (A.5).

An extension of $\kappa_{b v}$ into the interior of the rectangle $D$ can be achieved by making use of the particular structure (3.26) required for the projectors $P(y)$ as well as the assumed regularity of its matrix entries. This allows us to find functions $a, b \in C^{3}\left(D_{0}\right)$, where $D_{0}$ is an open domain containing $\bar{D}$, and define

$$
\tilde{\mathcal{P}}(x, y):=\left(\begin{array}{cc}
a(x, y) & b(x, y) \\
b(l-x, y) & a(l-x, y)
\end{array}\right)
$$

in such a way that $\tilde{P}(y)=\tilde{\mathcal{P}}(0, y)$. We noted in Section 3 that when rk $\tilde{P} \in\{0,2\}$, the only options for $\tilde{P}(y)$ are zero or $\mathbb{1}_{2}$. When $\operatorname{rk} \tilde{P}=0$ we hence can choose $a(x, y)=0=b(x, y)$ for all $(x, y) \in D_{0}$, and when $\mathrm{rk} \tilde{P}=2$ a corresponding choice would be $a(x, y)=1$ and $b(x, y)=0$ for all $(x, y) \in D_{0}$. If $\operatorname{rk} \tilde{P}=1$ and $\tilde{P}(y)$ is of the form (3.27) we have to pick a function $a \in C^{3}\left(D_{0}\right)$ that interpolates between $\beta(y)$ at $x=0$ and $1-\beta(y)$ at $x=l$, and a function $b \in C^{3}\left(D_{0}\right)$ that interpolates between $\bar{\gamma}(y)$ at $x=0$ and $\gamma(y)$ at $x=l$.

Due to the required regularity of the respective functions the following Taylor expansions,

$$
\tilde{\mathcal{P}}(x, y \pm h)=\tilde{\mathcal{P}}(x, y) \pm h \tilde{\mathcal{P}}_{y}(x, y)+h^{2} \tilde{\mathcal{P}}_{R_{2}}^{ \pm}(x, y ; h)
$$

and

$$
\tau^{2}(y-h)=\tau^{2}(y)-h \tau_{R_{1}}^{2}(y ; h),
$$

hold with remainder terms that are of class $C^{1}$ in the variable $y$ and are bounded in $h$. Using these expansions in (A.18) yields

$$
\begin{aligned}
\tilde{\kappa}_{b v}(y)= & \tau^{2}(y) \tilde{P}_{y}(y)\left(\frac{\tilde{\phi}_{b v}(y+h)-\tilde{\phi}_{b v}(y-h)}{h}\right)+\tau_{R_{1}}^{2}(y ; h) \tilde{P}_{y}(y) \tilde{\phi}_{b v}(y-h) \\
& +\tau^{2}(y) \tilde{P}_{R_{2}}^{+}(y ; h) \tilde{\phi}_{b v}(y+h)+\tau^{2}(y-h) \tilde{P}_{R_{2}}^{-}(y ; h) \tilde{\phi}_{b v}(y-h) .
\end{aligned}
$$


We then define the function

$$
\begin{aligned}
\kappa(x, y):= & \tau^{2}(y)\left(a_{y}(x, y) \frac{\phi(x, y+h)-\phi(x, y-h)}{h}\right. \\
& \left.\quad+b_{y}(x, y) \frac{\phi(l-x, y+h)-\phi(l-x, y-h)}{h}\right) \\
& +\tau_{R_{1}}^{2}(y ; h)\left(a_{y}(x, y) \phi(x, y-h)+b_{y}(x, y) \phi(l-x, y-h)\right) \\
& +\tau^{2}(y)\left(a_{R_{2}}^{+}(x, y ; h) \phi(x, y+h)+b_{R_{2}}^{+}(x, y ; h) \phi(l-x, y+h)\right) \\
& +\tau^{2}(y-h)\left(a_{R_{2}}^{-}(x, y ; h) \phi(x, y-h)+b_{R_{2}}^{-}(x, y ; h) \phi(l-x, y-h)\right),
\end{aligned}
$$

whose boundary values indeed satisfy (A.16). The regularity of the functions involved implies that $\kappa \in H^{1}(D)$ and, thus, $\|\kappa\|_{L^{2}(D)}$ and $\|\kappa\|_{H^{1}(D)}$ are finite. Moreover, since $\phi \in H^{1}(D)$ and

$$
\frac{\phi(x, y+h)-\phi(x, y-h)}{h}=D_{y}^{+h} \phi(x, y)+D_{y}^{-h} \phi(x, y)
$$

Theorem A.2 implies that $\|\kappa\|_{L^{2}(D)}$ has an $h$-independent upper bound. In the same way the second bound in (A.15) follows from (A.23) and (A.24).

Next we estimate the fifth term on the right-hand side of (A.14). We use the selfadjointness of $\Lambda$ and perform an integration by parts (A.4) as well as employing the product rule (A.3) to obtain

$$
\begin{aligned}
\left\langle\phi_{b v}, \Lambda\left(D_{y}^{-h} \tau^{2} D_{y}^{+h} \phi\right)_{b v}\right\rangle_{L^{2}(0, l) \otimes \mathbb{C}^{4}}= & -\int_{0}^{l}\left\langle L(y+h)\left(\tau D_{y}^{+h} \phi_{b v}\right)(y),\left(\tau D_{y}^{+h} \phi_{b v}\right)(y)\right\rangle_{\mathbb{C}^{4}} \mathrm{~d} y \\
& -\int_{0}^{l}\left\langle\left(\tau\left(D_{y}^{+h} L\right) \phi_{b v}\right)(y),\left(\tau D_{y}^{+h} \phi_{b v}\right)(y)\right\rangle_{\mathbb{C}^{4}} \mathrm{~d} y .
\end{aligned}
$$

Noting that $L$ is supposed to be bounded and Lipschitz continuous, the right hand side can be estimated from above in absolute value by

$$
d_{1}\left\|\tau D_{y}^{+h} \phi_{b v}\right\|_{L^{2}(0, l) \otimes \mathbb{C}^{4}}^{2}+d_{2}\left\|\phi_{b v}\right\|_{L^{2}(0, l) \otimes \mathbb{C}^{4}}\left\|\tau D_{y}^{+h} \phi_{b v}\right\|_{L^{2}(0, l) \otimes \mathbb{C}^{4}},
$$

with suitable constants $d_{j}>0$. Estimating further, we apply (3.8) to the first term and (3.12) to the second and obtain the bound

$$
4 d_{1}\left(\frac{2}{\epsilon_{4}}\left\|\tau D_{y}^{+h} \phi\right\|_{L^{2}(D)}^{2}+\epsilon_{4}\left\|\nabla\left(\tau D_{y}^{+h} \phi\right)\right\|_{L^{2}(D)}^{2}\right)+d_{3}\left\|\phi_{b v}\right\|_{L^{2}(0, l) \otimes \mathbb{C}^{4}}\left\|\tau D_{y}^{+h} \phi\right\|_{H^{1}(D)},
$$

where $\epsilon_{4}>0$ is sufficiently small. Eventually, again using (3.8), this can be further bounded by

$$
d_{4}+d_{5} \epsilon_{5}\left\|\tau D_{y}^{+h} \nabla \phi\right\|_{L^{2}(D)}^{2}, \quad \text { where } d_{j}>0 .
$$

It remains to estimate the last-but-one term on the right-hand side of (A.14),

$$
\left\|D_{y}^{-h} \tau^{2} D_{y}^{+h} \phi\right\|_{L^{2}(D)} \leq\left\|\partial_{y}\left(\tau^{2} D_{y}^{+h} \phi\right)\right\|_{L^{2}(D)} \leq d_{6}+d_{7}\left\|\tau^{2} D_{y}^{+h} \nabla \phi\right\|_{L^{2}(D)} .
$$


The last term on the right-hand side can be estimated with the help of (A.13), and using that $\tau^{2} \leq 1$

$$
\left\|D_{y}^{-h} \tau^{2} D_{y}^{+h} \phi\right\|_{L^{2}(D)} \leq c_{6}\left(\epsilon_{6}\right)+d_{8} \epsilon_{6}\left\|\tau D_{y}^{+h} \nabla \phi\right\|_{L^{2}(D)}^{2} .
$$

We now collect all bounds for the terms on the right-hand side of (A.14) and subtract all contributions of the form $\epsilon_{j}\left\|\tau D_{y}^{+h} \nabla \phi\right\|_{L^{2}(D)}^{2}$. By choosing $\epsilon_{1}, \ldots, \epsilon_{6}$ sufficiently small we finally obtain the bound (A.5). By applying Lemma A.1 to $\nabla \phi$ on the domain $D^{\prime}=$ $[0, l] \times[\epsilon, l-\epsilon]$ we conclude that $\phi_{x y}$ and $\phi_{y y}$ are in $H^{2}\left(D^{\prime}\right)$. Since $\phi_{x x}+\phi_{y y}=\Delta_{2} \phi$ is known to be in $L^{2}(D)$ we conclude that $\phi \in H^{2}\left(D^{\prime}\right)$. The same argument can now be repeated on a domain $D^{\prime \prime}=[\epsilon, l-\epsilon] \times[0, l]$ so that, indeed, $\phi$ has $H^{2}$-regularity away from small neighbourhoods of the corners of the rectangle $D$.

As the condition imposed on $P$ implies that close to the corners either Dirichlet or Neumann or mixed Dirichlet-Neumann boundary conditions are imposed, regularity of $\phi$ in neighbourhoods of the corners follows from standard results (see, e.g., [Neč67, Dau88]).

\section{References}

[BE09] J. Bolte and S. Endres, The trace formula for quantum graphs with general self-adjoint boundary conditions, Ann. H. Poincare 10 (2009), 189-223.

[BGS84] O. Bohigas, M. J. Giannoni, and C. Schmit, Characterization of chaotic quantum spectra and universality of level fluctuation laws, Phys. Rev. Lett. 52 (1984), 1-4.

[Dau88] M. Dauge, Elliptic boundary value problems on corner domains, Lecture Notes in Mathematics, vol. 1341, Springer-Verlag, Berlin, 1988, Smoothness and asymptotics of solutions.

[Dob05] M. Dobrowolski, Angewandte Funktionalanalysis: Funktionalanalysis, SobolevRäume und Elliptische Differentialgleichungen, Springer-Verlag, Berlin, 2005.

$\left[\mathrm{EKK}^{+}\right.$08] P. Exner, J. P. Keating, P. Kuchment, T. Sunada, and A. Teplyaev (eds.), Analysis on graphs and its applications, Proceedings of Symposia in Pure Mathematics, vol. 77, American Mathematical Society, Providence, RI, 2008, Papers from the program held in Cambridge, January 8-June 29, 2007.

[GS06] S. Gnutzman and U. Smilansky, Quantum graphs: application to quantum chaos and universal spectral statistics, Adv. in Phys. 55 (2006), 527 - 625.

[GT83] D. Gilbarg and N. S. Trudinger, Elliptic partial differential equations of second order, second ed., Grundlehren der Mathematischen Wissenschaften, vol. 224, Springer-Verlag, Berlin, 1983. 
[Har07] M. Harmer, Two particles on a star graph. I, Russ. J. Math. Phys. 14 (2007), $435-439$.

[Har08] _ Two particles on a star graph. II, Russ. J. Math. Phys. 15 (2008), $473-480$.

[HKR11] J. M. Harrison, J. P. Keating, and J. M. Robbins, Quantum statistics on graphs, Proc. R. Soc. Lond. Ser. A 467 (2011), 212-233.

[Kar31] J. Karamata, Neuer Beweis und Verallgemeinerung einiger Tauberian-Sätze, Math. Z. 33 (1931), 294-299.

[Kat66] T. Kato, Perturbation theory for linear operators, Die Grundlehren der mathematischen Wissenschaften, Band 132, Springer-Verlag New York, Inc., New York, 1966.

[KS99a] V. Kostrykin and R. Schrader, Kirchhoff's rule for quantum wires, J. Phys. A: Math. Gen. 32 (1999), 595-630.

[KS99b] T. Kottos and U. Smilansky, Periodic orbit theory and spectral statistics for quantum graphs, Ann. Phys. (NY) 274 (1999), 76-124.

[Kuc04] P. Kuchment, Quantum graphs. I. Some basic structures, Waves Random Media 14 (2004), S107-S128.

[Neč67] J. Nečas, Les méthodes directes en théorie des équations elliptiques, Masson et Cie, Éditeurs, Paris, 1967.

[RS53] K. Ruedenberg and C. W. Scherr, Free-electron network model for conjugated systems. I. Theory, J. Chem. Phys. 21 (1953), 1565-1581.

[RS72] M. Reed and B. Simon, Methods of modern mathematical physics. I. Functional analysis, Academic Press, New York, 1972.

[RS78] Methods of modern mathematical physics. IV. Analysis of operators, Academic Press, New York, 1978.

[RS79] - Methods of modern mathematical physics. III, Academic Press, New York, 1979, Scattering theory.

[Sho77] R. E. Showalter, Hilbert space methods for partial differential equations, Pitman, London, 1977, Monographs and Studies in Mathematics, Vol. 1. 